\title{
A theory for coupled lithium insertion and viscoplastic flow in amorphous anode materials for Li-ion batteries
}

\author{
Afsar Bagheri ${ }^{\mathrm{a}}$, Jamal Arghavani ${ }^{\mathrm{a}}$, Reza Naghdabadi ${ }^{\mathrm{a}, \mathrm{b}}$, Laurence Brassart ${ }^{\mathrm{c}, \mathrm{d}, *}$ \\ ${ }^{a}$ Department of Mechanical Engineering, Sharif University of Technology, Tehran, Iran \\ ${ }^{b}$ Institute for Nano-Science and Technology, Sharif University of Technology, Tehran, Iran \\ ${ }^{c}$ Department of Engineering Science, University of Oxford, Oxford, OX1 3PJ, United Kingdom \\ ${ }^{d}$ Department of Materials Science and Engineering, Monash University, Clayton, VIC 3800, Australia
}

\begin{abstract}
Amorphous lithium metal alloys $\left(\mathrm{Li}_{x} \mathrm{M}\right.$, with $\left.\mathrm{M}=\mathrm{Si}, \mathrm{Ge}, \mathrm{Sn}, \ldots\right)$ are attractive anode materials for lithium-ion batteries owing to their high energy-storage capacity and safety characteristics. However, repeated insertion of lithium often leads to chemo-mechanical degradation of the alloy, which can severely reduce the battery capacity and cycle life. Better understanding of the chemo-mechanical response of lithium alloys is needed to guide the design of damage-resistant anode microstructures. In this work, we propose a constitutive theory that couples large, viscoplastic deformations to the insertion and extraction of lithium in amorphous electrode materials. The theory relies on the concept of Shear Transformation Zone as carrier of plastic flow in the amorphous material, and accounts for microstructural evolution via an internal "free volume" variable. The model is used to gain insight into several features of the plasticity of amorphous alloys during lithiation, including rate-dependency, pressure-dependency, and structural evolution. Model predictions are also compared to experimental data for amorphous silicon.

Keywords: Lithiation; Plasticity; Silicon; Free volume; Shear Transformation Zones
\end{abstract}

\section{Introduction}

Lithium metal alloys $\left(\mathrm{Li}_{x} \mathrm{M}\right.$, with $\left.\mathrm{M}=\mathrm{Si}, \mathrm{Ge}, \mathrm{Sn}, \ldots\right)$ are promising anode materials for 3 lithium-ion batteries owing to their high energy-storage capacity (Scrosati and Garche, 4 2010; Zhang, 2011). For example, the theoretical specific capacity of silicon is $4200 \mathrm{mAh} / \mathrm{g}$, 5 about ten times higher than the capacity of conventional graphite anodes. However, inser6 tion of lithium into the host alloy results in large volume change (up to $300 \%$ for silicon),

\footnotetext{
*Corresponding author. Email: laurence.brassart@eng.ox.ac.uk
} 
which can lead to chemo-mechanical degradation of the electrode and rapid capacity decay of the battery (Deshpande et al., 2012; Nadimpalli et al., 2012; Zhang et al., 2017). Predicting the stresses generated in lithium metal alloys is important to guide the design of anodes with high capacity and long cycle life. In this work, we propose a constitutive model for large deformations coupled to lithium insertion in amorphous lithium alloys, building on previous theories for metallic glasses. We focus on amorphous lithium-silicon alloy as a model system, which has been intensively studied in recent years. However, the proposed constitutive framework is applicable to other amorphous alloys.

The deformation behaviour of a-LiSi displays a number of interesting features during lithiation-delithiation cycles:

1. Insertion of lithium is accommodated by large plastic deformations. Several authors have measured the stresses generated during cyclic lithiation of amorphous silicon thin films (Sethuraman et al., 2010a,b; Soni et al., 2012; Pharr et al., 2013, 2014; Bucci et al., 2014). For a constant lithiation rate, stress-concentration curves exhibit an elastic-plastic transition, with yield stress decreasing as lithium concentration increases. The stress-limiting role of plasticity has been recognised as a key element in preventing fracture of small-size electrodes (Zhao et al., 2011b). The composition dependence of the elastic modulus and hardness has been confirmed by nanoindentation studies (Hertzberg et al., 2011; Berla et al., 2015; Sitinamaluwa et al., 2017; de Vasconcelos et al., 2017), and further investigated using atomistic simulations (Shenoy et al., 2010; Zhao et al., 2011c; Cui et al., 2012). Recent molecular dynamics studies suggest that plastic deformation of a-LiSi involves local rearrangements of atoms in Shear Transformation Zones (STZs) involving mostly lithium atoms (Yan et al., 2017; Darbaniyan et al., 2020). STZs have been recognised as the plasticity carriers in other amorphous metals (Schuh et al., 2007).

2. Plastic flow of a-LiSi is rate-dependent. Lithiation-rate dependence was observed in thin film experiments, although reported measures of rate sensitivity are somewhat contradictory. For example, Pharr et al. (2014) carried out rate-jumps experiments on silicon thin films and observed a marked increase in flow stress following an increase in lithiation rate. The relationship between strain rate and stress was well described by a power law with stress exponent in the range 2.6-4. In contrast, Bucci et al. (2014) conducted lithiation/deliathion steps at varying rates and found 
a much larger stress-exponent value (about 50), suggesting a much weaker strainrate sensitivity. Recent operando nanoindentation creep tests confirmed the ratedependent flow behaviour over a broad range of compositions, with a sharp increase in strain-rate sensitivity of a-Si upon the start of lithiation, followed by a constant strain-rate sensitivity for compositions over $\operatorname{Li}_{0.5} \mathrm{Si}$ (de Vasconcelos et al., 2017). Strain-rate dependency is also consistent with the STZ-mediated plasticity picture.

3. Plastic flow of a-LiSi is pressure-dependent. Some (but not all) thin film experiments suggest a tension-compression asymmetry in the flow stress of a-LiSi (Sethuraman et al., 2010a; Bucci et al., 2014). Pressure-dependent plastic flow was confirmed in atomistic simulations (Zhao et al., 2013; Fan et al., 2013; Sitinamaluwa et al., 2017). Pressure-dependency has also been reported in the context of the plasticity of bulk metallic glasses (Schuh and Lund, 2003; Schuh et al., 2007).

4. Cyclic lithiation induces microstructural evolution and permanent volume change (Ghassemi et al., 2011; McDowell et al., 2013; Chen et al., 2016; Jerliu et al., 2017). For example, in-situ TEM studies showed that a-Si nanostructures have a larger volume (up to 25\%) following a complete lithiation-delithiation cycle (Ghassemi et al., 2011; McDowell et al., 2013), which was attributed either to trapped Li or to the formation of nanovoids during delithiation. Using STEM, Wetjen et al. (2018) confirmed that dealloying reactions induce severe morphological changes in Si nanoparticles during lithiation-delithiation cycles. These morphological changes include the transformation of solid Si particles into porous networks with high surface area, as well as a permanent volume change of up to $700 \%$ after 60 cycles. The formation of a spongelike network due to dealloying reactions during electrochemical cycles was also reported in Li-Ge alloys (Liu et al., 2011) as well as in Li-Sn alloys (Chao et al., 2011; Chen and Sieradzki, 2013). Structural evolution during lithiation-delithiation cycles was also observed in atomistic simulations, including nanopore formation (Zhao et al., 2012) and increased disorder in the microstructure (Sitinamaluwa et al., 2017). Kim et al. (2017) simulated the delithiation process using molecular dynamics simulations and found that the density of delithiated silicon was always smaller than the density of equilibrated structure of the same composition. While nanopores formed at slow delithiation rates can be partially recovered by structural relaxation, nanopores formed at high rates have no time to shrink and 
lead to significant volume change.

Several constitutive theories have been proposed in order to capture the deformation behaviour of amorphous silicon during lithiation-delithiation cycles. Phenomenological models have relied on $\mathrm{J}_{2}$ (visco)plasticity, wherein chemo-mechanical softening is captured via composition-dependent material parameters, including the elastic moduli and the yield stress (Zhao et al., 2011a; Brassart et al., 2013; Bower et al., 2011; Cui et al., 2012; Pharr et al., 2014; Bucci et al., 2014; Di Leo et al., 2015; Karami and Nayebi, 2020). In addition, a defect-based viscoplastic model has been proposed, recently (Li et al., 2019a,b). However, using first-principles calculations, Zhao et al. (2012) showed that chemical reaction enables plastic flow at a level of stress significantly lower than under mechanical loading alone. This observation suggests a coupling between insertion of lithium and stress that cannot be captured using conventional plasticity theory with composition-dependent parameters. Motivated by this observation, Brassart and Suo $(2012,2013)$ developed a phenomenological theory of "reactive flow", according to which plastic flow occurs when a combination of deviatoric stresses and chemical potential reaches a critical value. Levitas and Attariani $(2013,2014)$ adopted a different point of view and postulated that lithiation-induced softening is due to anisotropic swelling under deviatoric stress, rather than conventional plasticity. Both approaches qualitatively reproduce the observations of Zhao et al. (2012). However, neither of these models explicitly rely on micromechanisms of deformation in the amorphous host. Also, they do not account for microstructure evolution during lithiation-delithiation cycles. The latter limitation was addressed by Bower et al. (2015), who proposed a thermodynamic theory for lithium insertion and transport in amorphous silicon by extending the Larché-Cahn model (Larché and Cahn, 1978) to account for irreversible distortion of the random lattice and for the creation and annihilation of unoccupied atomic sites. The model predicts irreversible volume change after lithiation cycles, as well as a tension-compression asymmetry in flow stress. The model is sophisticated and requires keeping track of $\mathrm{Si}, \mathrm{Li}$ and atomic sites concentrations. Khosrownejad and Curtin (2016) proposed an alternative approach whereby the microstructural state, or structural disorder of the system is described by an internal variable. Using molecular dynamics simulations, these authors showed that structures delithiated at a fast rate have an excess energy as compared to a fully-relaxed structure of the same composition. The excess energy (associated with structural disorder) in turn enables flow at a lower 
stress. These authors developed a constitutive theory assuming that the excess energy evolution has three contributions: insertion/extraction of lithium, STZ-mediated plastic flow and thermally-activated structural relaxation. However, the model does not predict irreversible volume change nor pressure-dependency.

Recently, Li et al. (2020a) developed a free volume-based viscoplastic model for amorphous silicon electrodes. Their model was built on the physical concept of individual atomic jumps rather than STZs involving the collective motion of several atoms. This contribution analysed stress, deformation and concentration in an amorphous thin-film Si electrode on a rigid substrate. However, the effect of pressure dependency and structural evolution was not investigated. In another contribution from the same group, the coupling effects of self-limiting lithiation, reaction front evolution and free volume evolution on the stress was investigated in amorphous wire-based electrodes (Li et al., 2020b). Regarding pressure-dependency, an analysis of deformation and failure of electrode with layered structure was proposed by Zhu et al. (2019), where the electrode response was described by the Drucker-Prager/Cap plasticity model.

In this work, we propose a physically-based constitutive model for amorphous electrode materials that can describe rate-dependency, pressure-dependency and structural evolution during lithiation-deliathiation cycles. Following Khosrownejad and Curtin (2016), we describe the state of the system at a given composition by an internal variable $\xi$ describing the deviation of the structure from its fully-relaxed configuration of lowest energy. We assume the following three contributions to the evolution of the structural disorder $\xi:$ 1) insertion/extraction of lithium, 2) STZ-mediated plastic flow and 3) structural relaxation. The latter is driven by the excess energy associated with structural disorder. Different from Khosrownejad and Curtin (2016), we associate inelastic volumetric strain to structural disorder evolution. It then follows from thermodynamics that the mean stress contributes to the driving force for structural disorder creation and annihilation. We propose a yield surface and flow rules that couple the deviatoric stresses, mean stress and driving force for structural relaxation. The viscoplastic flow rule is based on STZ dynamics and also includes a pressure-dependency. The model is written in a consistent thermodynamic framework that identifies the driving forces for the various kinetic processes and ensures that kinetic models satisfy a thermodynamic inequality. To focus on the essential ideas, we focus on homogeneous states, and use a finite strain formulation in 
principal stretches. We illustrate the model capabilities in several examples and compare model predictions to available experimental data for thin films.

The paper is organised as follows. Section 2 presents the constitutive model in a thermodynamically-consistent framework. Section 3 illustrates the model capability under several loading conditions, and explores the effects of material parameters. In Section 4, model predictions are compared to experimental data from the literature for the cyclic lithiation of a thin film electrode.

\section{Constitutive theory}

\subsection{Kinematics}

We consider a small piece of amorphous electrode material evolving through a sequence of homogeneous states. In the reference state, the piece is stress-free and lithium-free. Following Khosrownejad and Curtin (2016), we describe the structural disorder of the amorphous material with an internal variable $\xi$, which quantifies the excess energy of the system relative to a hypothetical, fully-relaxed state with minimum of free energy, corresponding to $\xi=0$. The precise relationship between the internal variable and the excess energy will be described in Section 2.3. In the reference state, the internal variable is $\xi_{0}$. In the current state at a particular time, subject to applied stresses and in contact with a reservoir of lithium, the stretches of the piece are $\lambda_{1}, \lambda_{2}$ and $\lambda_{3}$, the concentration of lithium is $C$ (number of $\mathrm{Li}$ atoms in the current state per unit reference volume) and the structural disorder variable is $\xi$. We define $J=\lambda_{1} \lambda_{2} \lambda_{3}>0$ the ratio of volume of the piece in the current state to that in the reference state.

We adopt the classical approach in finite-strain plasticity coupled to species insertion and decompose the stretches into elastic, inelastic (plastic) and chemical stretches (Zhao et al., 2011a; Bower et al., 2011; Di Leo et al., 2015):

$$
\lambda_{k}=\lambda_{k}^{e} \lambda_{k}^{p} \lambda_{k}^{c}, \quad k=1,2,3(\text { no sum on } k) .
$$

The elastic stretches $\lambda_{k}^{e}$ correspond to a distortion of atomic bonds with no rearrangements of atoms. The inelastic stretches $\lambda_{k}^{p}$ represent deformations due to rearrangements of atoms at a fixed composition. These rearrangements include inelastic deformations by STZ activation and dilatation due to structural disorder evolution. The chemical stretches $\lambda_{k}^{c}$ represent deformations due to insertion of lithium. We define $J^{e}=\lambda_{1}^{e} \lambda_{2}^{e} \lambda_{3}^{e}, J^{p}=$ 
$\lambda_{1}^{p} \lambda_{2}^{p} \lambda_{3}^{p}$, and $J^{c}=\lambda_{1}^{c} \lambda_{2}^{c} \lambda_{3}^{c}$, so that

$$
J=J^{e} J^{p} J^{c} .
$$

The chemical volume change is related to the lithium concentration by the following relation:

$$
J^{c}=1+\Omega C,
$$

where $\Omega$ is the volume per lithium atom and is assumed constant for simplicity. We further assume that deformation due to insertion of lithium is isotropic:

$$
\lambda_{1}^{c}=\lambda_{2}^{c}=\lambda_{3}^{c}=(1+\Omega C)^{1 / 3} .
$$

We define the elastic, plastic and chemical logarithmic strains respectively as $\varepsilon_{k}^{e}=$ $\log \lambda_{k}^{e}, \varepsilon_{k}^{p}=\log \lambda_{k}^{p}$ and $\varepsilon_{k}^{c}=\log \lambda_{k}^{c}$. The volumetric chemical strain is then given by: $\theta^{c}=\varepsilon_{1}^{c}+\varepsilon_{2}^{c}+\varepsilon_{3}^{c}=\log J^{c}$. From (3), we have the identity:

$$
\dot{\theta}^{c}=\frac{\dot{J}^{c}}{J^{c}}=\frac{\Omega}{J^{c}} \dot{C}
$$

where $\Omega / J^{c}$ represents the reference volume per lithium atom. Likewise, the volumetric plastic strain is given by $\theta^{p}=\varepsilon_{1}^{p}+\varepsilon_{2}^{p}+\varepsilon_{3}^{p}=\log J^{p}$. We assume that evolution of structural disorder is accompanied by inelastic volume change, and introduce the following coupling:

$$
\dot{\theta}^{p}=\frac{\dot{J}^{p}}{J^{p}}=\alpha \dot{\xi}
$$

where $\alpha$ is a dimensionless dilatation parameter that in general depends on concentration and free volume. Here, for simplicity we choose $\alpha=1$. The inelastic volume change and free volume are then one-to-one related by $J^{p}=\exp \left(\xi-\xi_{0}\right)$. The coupling (6) identifies the structural variable $\xi$ with a free volume variable, similar to previous dilatant amorphous plasticity theories (Anand and Su, 2005; Thamburaja and Ekambaram, 2007).

Remark 1. In the literature, free volume concentration has been defined as the excess volume relative to the volume of the sample in its dense, fully-relaxed state (Huang et al., 2002; Yang et al., 2006):

$$
\xi=\frac{V_{c}-V_{d}}{V_{d}},
$$

where $V_{c}$ and $V_{d}$ are the sample volume in the current and dense state, respectively (assuming no stress and constant composition). According to this definition of free volume 
concentration, the inelastic volume change is then given by $J^{p}=(1+\xi) /\left(1+\xi_{0}\right)$, which corresponds to the following evolution equation:

$$
\dot{\theta}^{p}=\frac{\dot{\xi}}{(1+\xi)} .
$$

The latter evolution equation is recovered by setting $\alpha=(1+\xi)^{-1}$ in Eq. (6). Therefore, the choice $\alpha=1$ is not strictly equivalent to definition (7) of free volume. However, the difference is expected to be small for small values of $\xi$.

Free volume is probably the most widely used internal variable in phenomenological theories of plasticity in amorphous materials (Spaepen, 1977; Argon, 1979; Falk and Langer, 1998). It is however not the only possible choice, and other internal variables have been proposed to describe the structural state of amorphous materials, such as effective temperature (Langer, 2004), liquidlike fraction (Demkowicz and Argon, 2005), configurational potential energy function (Johnson et al., 2007) or excess energy (Khosrownejad and Curtin, 2016). Ideally, the chosen internal variable should be accessible in experimental measurements or atomistic simulations. The determination of a suitable order parameter that can be linked to flow behaviour in metallic or network glasses is still a matter of debate (Ma, 2015; Ding et al., 2016; Fan et al., 2017; Zhu et al., 2017, 2018), which we do not seek to address here. In the following, we will refer to $\xi$ as "free volume" for simplicity.

Following Khosrownejad and Curtin (2016), we assume that the rate of free volume, $\dot{\xi}$, has three contributions: 1) insertion or extraction of lithium $\left.\left(\dot{\xi}_{c}\right), 2\right)$ STZ activation under stress $\left(\dot{\xi}_{\sigma}\right)$, and 3) structural relaxation $\left(\dot{\xi}_{r}\right)$ :

$$
\dot{\xi}=\dot{\xi}_{c}+\dot{\xi}_{\sigma}+\dot{\xi}_{r}
$$

The contribution of lithium insertion/extraction to structural disorder is taken of the following form:

$$
\dot{\xi}_{c}=\beta\left|\dot{\theta}^{c}\right|=\beta \frac{\Omega}{J^{c}}|\dot{C}|,
$$

where $\beta$ is a dimensionless number that takes a different value during lithiation and delithiation:

$$
\beta=\left\{\begin{array}{ll}
\beta_{l} & (\dot{C}>0) \\
\beta_{d} & (\dot{C}<0)
\end{array} .\right.
$$




\subsection{Thermodynamics}

Let $P_{1}, P_{2}$ and $P_{3}$ be the three principal nominal stresses (force per unit area in the reference configuration), $\mu$ be the chemical potential of lithium in the reservoir, and $W$ be the Helmholtz free energy of the piece (energy per unit reference volume). Thermodynamics requires that the power of the external mechanical and chemical loads should not be smaller than the change in Helmholtz free energy of the piece:

$$
P_{1} \dot{\lambda}_{1}+P_{2} \dot{\lambda}_{2}+P_{3} \dot{\lambda}_{3}+\mu \dot{C}-\dot{W} \geq 0
$$

Introduce the Cauchy stresses $\sigma_{1}=P_{1} / \lambda_{2} \lambda_{3}, \sigma_{2}=P_{2} / \lambda_{1} \lambda_{3}$ and $\sigma_{3}=P_{3} / \lambda_{1} \lambda_{2}$ (force per unit area in the current configuration), and define the mean stress $\sigma_{m}=\left(\sigma_{1}+\sigma_{2}+\sigma_{3}\right) / 3$. Using decomposition (1) of the stretches together with the definition of the logarithmic strains and equation (5), the inequality becomes:

$$
\begin{array}{r}
J\left(\sigma_{1} \dot{\varepsilon}_{1}^{e}+\sigma_{2} \dot{\varepsilon}_{2}^{e}+\sigma_{3} \dot{\varepsilon}_{3}^{e}\right)+J\left(s_{1} \dot{e}_{1}^{p}+s_{2} \dot{e}_{2}^{p}+s_{3} \dot{e}_{3}^{p}\right) \\
+J \sigma_{m} \dot{\theta}^{p}+J \sigma_{m} \frac{\Omega}{J^{c}} \dot{C}+\mu \dot{C}-\dot{W} \geq 0
\end{array}
$$

where $e_{k}^{p}=\varepsilon_{k}^{p}-\theta^{p} / 3$ is the deviatoric plastic strain and $s_{k}=\sigma_{k}-\sigma_{m}$ the deviatoric stress. Using Eqs (6), (9) and (10), the inequality becomes:

$$
\begin{array}{r}
J\left(\sigma_{1} \dot{\varepsilon}_{1}^{e}+\sigma_{2} \dot{\varepsilon}_{2}^{e}+\sigma_{3} \dot{\varepsilon}_{3}^{e}\right)+J\left(s_{1} \dot{e}_{1}^{p}+s_{2} \dot{e}_{2}^{p}+s_{3} \dot{e}_{3}^{p}\right) \\
+\Omega^{\prime} \sigma_{m} \beta \operatorname{sign}(\dot{C}) \dot{C}+J \sigma_{m}\left(\dot{\xi}_{\sigma}+\dot{\xi}_{r}\right)+\Omega^{\prime} \sigma_{m} \dot{C}+\mu \dot{C}-\dot{W} \geq 0,
\end{array}
$$

where $\operatorname{sign}(x)$ is the sign function and $\Omega^{\prime} \equiv \Omega J / J^{c}$ represents the volume per lithium atom in the current configuration.

We assume that the Helmholtz-free energy of the host is a function of the elastic strains, lithium concentration and free volume:

$$
W=\hat{W}\left(\varepsilon_{1}^{e}, \varepsilon_{2}^{e}, \varepsilon_{3}^{e}, C, \xi\right)
$$

Introducing expression (15) of the free energy, the inequality becomes:

$$
\begin{aligned}
J\left(\sigma_{1}-\frac{1}{J} \frac{\partial \hat{W}}{\partial \varepsilon_{1}^{e}}\right) \dot{\varepsilon}_{1}^{e}+ & J\left(\sigma_{2}-\frac{1}{J} \frac{\partial \hat{W}}{\partial \varepsilon_{2}^{e}}\right) \dot{\varepsilon}_{2}^{e}+J\left(\sigma_{3}-\frac{1}{J} \frac{\partial \hat{W}}{\partial \varepsilon_{3}^{e}}\right) \dot{\varepsilon}_{3}^{e} \\
& +\left(\mu-\frac{\partial \hat{W}}{\partial C}+\Omega^{\prime} \sigma_{m}+\Omega^{\prime} \beta \operatorname{sign}(\dot{C}) \zeta\right) \dot{C} \\
+ & J\left(s_{1} \dot{e}_{1}^{p}+s_{2} \dot{e}_{2}^{p}+s_{3} \dot{e}_{e}^{p}\right)+J \zeta\left(\dot{\xi}_{\sigma}+\dot{\xi}_{r}\right) \geq 0,
\end{aligned}
$$


where $\zeta$ has the dimension of stress and is given by:

$$
\zeta=\sigma_{m}-\frac{1}{J} \frac{\partial \hat{W}}{\partial \xi} .
$$

Inequality (16) identifies $\zeta$ as the force conjugated to free volume evolution. The mean stress contributes positively to the driving force as a result of the volume change associated with free volume evolution. The second term is the driving force for structural relaxation due to the reduction in free energy. We assume that $\partial \hat{W} / \partial \xi \geq 0$, so that $\zeta<0$ when $\sigma_{m}<\frac{1}{J} \frac{\partial \hat{W}}{\partial \xi}$.

We assume that stresses are at all times in equilibrium with the elastic strains, which gives the state laws for the stress:

$$
\sigma_{1}=\frac{1}{J} \frac{\partial \hat{W}}{\partial \varepsilon_{1}^{e}}, \quad \sigma_{2}=\frac{1}{J} \frac{\partial \hat{W}}{\partial \varepsilon_{2}^{e}}, \quad \sigma_{3}=\frac{1}{J} \frac{\partial \hat{W}}{\partial \varepsilon_{3}^{e}} .
$$

We also assume equilibrium with respect to the insertion of lithium:

$$
\mu=\frac{\partial \hat{W}}{\partial C}-\Omega^{\prime} \sigma_{m}-\Omega^{\prime} \beta \operatorname{sign}(\dot{C}) \zeta .
$$

The first term represents the chemical driving force for lithium insertion, whereas the second term is a consequence of Eq. (3). The effects of this term on diffusion kinetics and stresses were recently investigated by Bagheri et al. (2019). The last term results from the coupling (10) between free volume evolution and insertion or extraction of lithium. Since excess disorder is created during both insertion and extraction, a positive $\zeta$ decreases the chemical potential when $\dot{C}>0$, favouring insertion of lithium. Conversely, a positive $\zeta$ increases the chemical potential when $\dot{C}<0$, favouring extraction of lithium.

Accounting for the state laws (18) and (19), the inequality reduces to:

$$
J\left(s_{1} \dot{e}_{1}^{p}+s_{2} \dot{e}_{2}^{p}+s_{3} \dot{e}_{3}^{p}\right)+J \zeta\left(\dot{\xi}_{\sigma}+\dot{\xi}_{r}\right) \geq 0 .
$$

The reduced thermodynamic inequality is satisfied by prescribing a kinetic model to coevolve the plastic deformation and structural disorder. A general kinetic model assumes that the rates of deviatoric plastic strain and structure disorder are functions of the thermodynamic driving forces:

$$
\dot{e}_{k}^{p}=\hat{\dot{e}}_{k}^{p}\left(s_{l}, \zeta\right), \quad \dot{\xi}_{\sigma}=\hat{\dot{\xi}}_{\sigma}\left(s_{l}, \zeta\right), \quad \dot{\xi}_{r}=\hat{\dot{\xi}}_{r}\left(s_{l}, \zeta\right), \quad(l=1,2,3) .
$$

The kinetic equations must satisfy the inequality for independent variations of the deviatoric plastic strain and structural disorder. 


\subsection{Free energy function}

Following Khosrownejad and Curtin (2016), we assume that the free energy function can be additively decomposed into chemical, elastic and excess energy parts:

$$
\hat{W}\left(\varepsilon_{k}^{e}, C, \xi\right)=W^{c}(C)+W^{e}\left(\varepsilon_{k}^{e}, C\right)+W^{x c}(C, \xi) .
$$

The chemical part is assumed of the following form:

$$
W^{c}(C)=\mu_{0} C+k_{B} T C_{\max }(\bar{c} \log \bar{c}+(1-\bar{c}) \log (1-\bar{c}))
$$

where $\bar{c}$ is a composition variable defined as the concentration divided by the maximum nominal concentration of lithium in the host: $\bar{c}=C / C_{\max }, \mu_{0}$ is a reference chemical potential, $k_{B}$ is Boltzmann's constant and $T$ the absolute temperature. This expression only accounts for the entropic contribution from mixing lithium atoms with the host atoms and neglects enthalpic conribution due to bonding between lithium and host atoms. A more elaborate description of the solution thermodynamics based on series representation of the enthalpic contribution to mixing has been proposed by Bucci et al. (2014), but has not been considered here for simplicity.

The elastic energy is taken of the following form:

$$
W^{e}\left(\varepsilon_{k}^{e}, C\right)=J^{c}\left[\frac{1}{2} \lambda(\bar{c})\left(\varepsilon_{1}^{e}+\varepsilon_{2}^{e}+\varepsilon_{3}^{e}\right)^{2}+G(\bar{c})\left(\left(\varepsilon_{1}^{e}\right)^{2}+\left(\varepsilon_{2}^{e}\right)^{2}+\left(\varepsilon_{3}^{e}\right)^{2}\right)\right],
$$

where $\lambda$ and $G$ are respectively the Lamé coefficient and the shear modulus, which depend on composition. They are related to Young's modulus and Poisson's ratio by the classical relations: $\lambda=\frac{E \nu}{(1+\nu)(1-2 \nu)}$ and $G=\frac{E}{2(1+\nu)}$. The elastic constants have units of energy per unit volume in the swollen configuration.

The last term $W^{x c}$ in Eq. (22) is the excess energy associated with free volume, or structural disorder. It represents the difference in free energy between the actual state and a (hypothetical) stress-free, fully-relaxed state (i.e. with $\xi=0$ ) at the same composition (Khosrownejad and Curtin, 2016). We adopt a simple quadratic expression:

$$
W^{x c}(C, \xi)=J^{c} \frac{k}{2} \xi^{2}
$$

where $k>0$ has the unit of energy per unit volume in the swollen configuration and is assumed constant for simplicity. For a given composition, Expression (25) thus establishes a mapping between the structural variable $\xi$ and the excess energy. 


$$
\sigma_{k}=\frac{J^{c}}{J}\left[2 G \varepsilon_{k}^{e}+\lambda\left(\varepsilon_{1}^{e}+\varepsilon_{2}^{e}+\varepsilon_{3}^{e}\right)\right],
$$

and the chemical potential (19) particularizes as:

$$
\begin{array}{r}
\mu=\mu_{0}+k_{B} T \log \left(\frac{C}{C_{\max }-C}\right)+\frac{\partial W^{e}}{\partial C}+\frac{\partial W^{x c}}{\partial C} \\
-\Omega^{\prime} \sigma_{m}-\Omega^{\prime} \beta \operatorname{sign}(\dot{C}) \zeta,
\end{array}
$$

where $\zeta$ is given by:

$$
\zeta=\sigma_{m}-k^{\prime} \xi
$$

where $k^{\prime} \equiv k J^{c} / J$ has units of energy per volume in the current configuration.

\subsection{Kinetic model}

Our kinetic model rests on the following physical picture: plastic strains results from thermally-activated microscopic rearrangements of atoms in STZs under an applied stress. Shear transformation in turn generates free volume via the contribution $\dot{\xi}_{\sigma}$, and is therefore also assumed to depend on $\zeta$, with positive (resp. negative) $\zeta$ promoting (resp. hindering) shear transformation. Mathematically, we couple deviatoric plastic deformation and free volume creation by inspiring from the Drucker-Prager model for pressure-dependent materials (Drucker and Prager, 1952). We postulate the existence of a yield function $f\left(s_{k}, \zeta\right)$ of the following form:

$$
f=\sigma_{e}+b \zeta
$$

where $\sigma_{e}$ is the von Mises equivalent stress and $b>0$ is a dimensionless parameter. The condition $f<0$ defines an elasticity domain in the three-dimensional space $\left(s_{k}, \zeta\right)$. Plastic flow can only occur when $\sigma_{e}>-b \zeta$. The threshold for flow is increased by a negative mean stress and by the driving force for structural relaxation, $\partial \hat{W} / \partial \xi$. Note that, in contrast to the classical Drucker-Prager model, we did not include a yield stress in expression (29). While a yield stress could easily be added to the model, we chose not to include it since the mechanistic origin of such a yield stress is not clear in the context of amorphous plasticity. In the context of the plasticity of amorphous metals described by the Drucker-Prager model, the parameter $b$ has been shown to govern the inclination of shear bands during plastic localisation, and takes value in the range $(0.045-0.205)$ (Zhao and Li, 2009). 
We construct flow rules by assuming that $\left(\dot{e}_{k}^{p}, \dot{\xi}_{\sigma}\right)$ is normal to the yield surface (associative plasticity):

$$
\begin{aligned}
& \dot{e}_{k}^{p}=\dot{p} \frac{\partial f}{\partial s_{k}}=\dot{p} \frac{3}{2} \frac{s_{k}}{\sigma_{e}} \\
& \dot{\xi}_{\sigma}=\dot{p} \frac{\partial f}{\partial \zeta}=b \dot{p},
\end{aligned}
$$

where $\dot{p}$ is the plastic multiplier, such that:

$$
\dot{p}=0 \quad \text { if } \quad f \leq 0, \quad \dot{p}>0 \quad \text { if } \quad f>0 .
$$

It is easily verified that the plastic multiplier is equal to the accumulated plastic strain rate: $\dot{p}=\left\{(2 / 3)\left(\left(\dot{e}_{1}^{p}\right)^{2}+\left(\dot{e}_{2}^{p}\right)^{2}+\left(\dot{e}_{3}^{p}\right)^{2}\right)\right\}^{1 / 2}$. The normality assumption was adopted for simplicity, and because it ensures positive dissipation. Alternatively, a non-associative plasticity model could have been used by selecting a flow potential distinct from the yield function in the flow rules (30)-(31). Consequences of the model (30)-(31) are the following. Deviatoric plastic strains are aligned with the deviatoric stresses (like in conventional $\mathrm{J}_{2}$ plasticity) and vanish under purely hydrostatic loading. Free volume creation due to mechanical loading, $\dot{\xi}_{\sigma}$, is directly proportional to the accumulated plastic strain rate, and therefore also vanish under purely hydrostatic loading. Free volume can however be created or annihilated via insertion/extraction of lithium $\left(\dot{\xi}_{c}\right)$ and structural relaxation $\left(\dot{\xi}_{r}\right)$.

In a rate-dependent plasticity model, the plastic multiplier is set by a viscoplastic function when $f \geq 0$. We inspire from a successful model for amorphous plasticity (Argon, 1979) and adopt the following expression:

$$
\dot{p}=2 \dot{p}_{0} \exp \left(-\frac{\Delta G}{k_{B} T}\right) \sinh \left(\frac{f V}{k_{B} T}\right) .
$$

This expression is obtained by considering STZ activation as a thermally-activated process with energy barrier $\Delta G$ (Eyring, 1936). $V$ is the apparent STZ volume (which includes the transformation strain) and $\dot{p}_{0}$ a parameter proportional to the attempt frequency. In the original theory (Eyring, 1936; Argon, 1979), the energy barrier for STZ transformation under shear is lessen by the work of the applied shear stress: $\tau V / k_{B} T$. In writing (33), we have extended Argon's model to multiaxial stress states and included the effect of pressure on the transformation kinetics. 
The energy barrier $\Delta G$ depends on the microstructural state and is therefore taken as a function of composition and free volume:

$$
\Delta G=\Delta G_{0}(\bar{c})+g(\xi),
$$

where $\Delta G_{0}(\bar{c})$ is the energy barrier at a given composition in the fully-relaxed state. Here we assume that the energy barrier decreases with composition as:

$$
\Delta G_{0}(\bar{c})=\Delta G_{\text {lith }}+\left(\Delta G_{\text {unlith }}-\Delta G_{\text {lith }}\right) \exp (-\bar{c} / n)
$$

where $\Delta G_{\text {unlith }}$ is the energy barrier for flow in the unlithiated host and $\Delta G_{\text {lith }}$ is the energy barrier in the fully-lithiated host. The parameter $n$ is a fitting parameter that controls the rate of decrease of the energy barrier. The function $g(\xi)$ in Eq. (34) describes the effect of free volume on the energy barrier and is chosen of the following form:

$$
g(\xi)=\frac{m}{\xi} k_{B} T
$$

where $m$ is a dimensionless parameter. Expression (36) together with (34) leads to a contribution of free volume to the flow equation (33) that is similar to free-volume theories (Cohen and Turnbull, 1959; Spaepen, 1977), where the parameter $m$ was identified as a geometric parameter related to the rearrangement of free volume during relaxation.

Free volume annihilation by structural relaxation is described by a simple linear kinetic model:

$$
\dot{\xi}_{r}=q \zeta
$$

where the kinetic parameter $q$ in general depends on composition and free volume. Assuming that more free volume allows faster relaxation, and noting that no relaxation can take place when the free volume is at its minimum value $\xi=0$, a simple expression for $q$ is:

$$
q=q_{0} \xi
$$

where $q_{0}$ is a positive constant. Inserting the kinetic model (30), (31), (32) and (37) into (20), it is readily verified that the inequality is satisfied.

\section{Numerical examples}

\subsection{Uniaxial loading}

We illustrate the model capability by first considering uniaxial loading at constant composition. In the initial configuration, the concentration is $C_{0}$, the disorder parameter 
is $\xi_{0}$, and the sample is stress free. The elastic constants are set as $E=67 \mathrm{GPa}$ and $\nu=0.3$, which are representative values for $\mathrm{Li}_{2} \mathrm{Si}$ (de Vasconcelos et al., 2017). We use $b=0.05$ in the Drucker-Prager yield criterion (29), which is within the range of values reported by Zhao and Li (2009). The energetic parameter $k$ is chosen such that the order of magnitude of the structural relaxation contribution to $\zeta$ (second term in Eq. (28)) is comparable or smaller to the contribution of the mean stress. Since the order of magnitude of mean stress is $\sim 10^{9} \mathrm{~Pa}, k$ is set equal to $5 \times 10^{10} \mathrm{~Pa}$. For the kinetic equation (33), we follow the work of Khosrownejad and Curtin (2016) and use $\dot{p}_{0}=5 \times 10^{8} \mathrm{~s}^{-1}$ for the frequency parameter and $V=100 \AA^{3}$ as apparent activation volume. Fixed composition leads to a constant value for $\Delta G_{0}$ in Eq. (34), which is chosen as $\Delta G_{0}=1.8 \times 10^{-19} \mathrm{~J}$, similar to the value proposed by Khosrownejad and Curtin (2016) when $x$ in $\operatorname{Li}_{x} \mathrm{Si}$ is equal to 2. The structural parameter $m$ in Eq. (36) is set to 0.01. Boltzmann's constant is $k_{B}=1.38 \times 10^{-23} \mathrm{~J} / \mathrm{K}$ and the absolute temperature is $T=300 \mathrm{~K}$. The kinetic parameter for structural relaxation, $q_{0}$, is set to $q_{0}=5 \times 10^{-12}(\mathrm{~Pa} \mathrm{~s})^{-1}$. Finally, the initial free volume is set equal to $\xi_{0}=0.001$. In the following, all parameters are kept at these values, unless otherwise indicated for the parametric study.

The evolution of stress and disorder during uniaxial loading at different applied strain rates are shown in Fig. 1. The linear part of stress-strain curves reveals that the initial response is mainly elastic, despite the absence of a formal yield stress in the model, cf. Eq. (29). During the pseudo-elastic part of the deformation, the dominant contribution to free volume evolution is the relaxation part, $\xi_{r}$. The stress then peaks and subsequently drops (post-yield softening), before reaching a stress plateau. The softening is attributed to the concurrent increase in free volume, which reduces the energy barrier for flow. Eventually, the disorder reaches a steady-state (e.g. the plateau of the blue curve in Fig. 1b), at which the creation and annihilation of disorder are equal and the material flows like a liquid. The time needed to reach steady-state depends on the applied strain rate, as well as on the material parameters. For example, at the largest applied strain rate considered here, a higher free volume creation rate compared to annihilation rate leads to a continuous increase in free volume and decrease of stress over the considered time scale (black curves in Fig. 1). Consequently, the model predicts increasing i) peak stress, ii) strain-softening and iii) steady-state stress with increasing rate of deformation, which is consistent with experimental observations for metallic glasses (De Hey et al., 1998; Lu et al., 2003). 


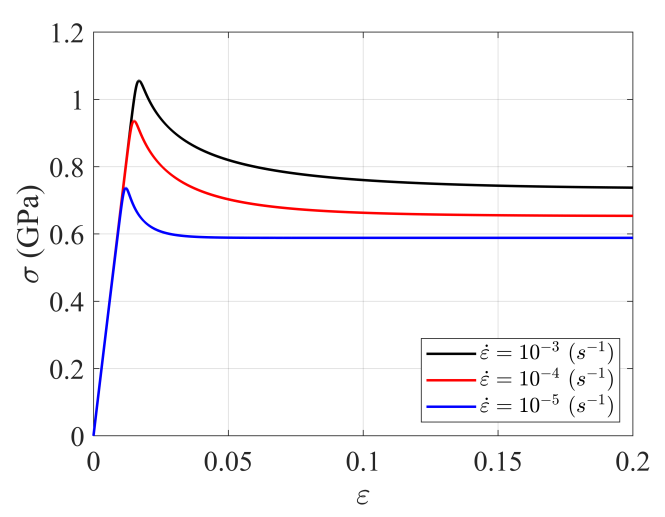

(a)

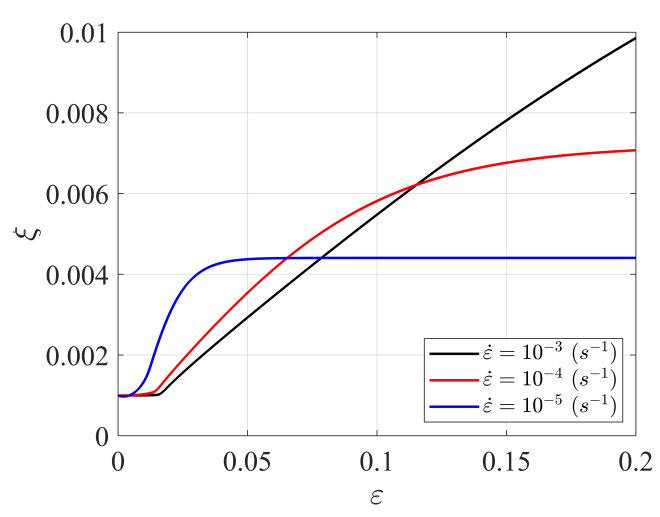

(b)

Figure 1: The evolution of a) stress and b) free volume in uniaxial tension at various strain rates.

The effect of energy barrier and apparent STZ volume on the evolution of stress and free volume is illustrated in Fig. 2 for a constant strain rate $\dot{\varepsilon}=10^{-5} \mathrm{~s}^{-1}$. The stress peak, steady-state stress and steady-state value of free volume all depend on $\Delta G_{0}$ and $V$. The effect of the energy barrier and STZ volume on the stress response can be understood in the following way. Under the condition of large stress $\left(f V \gg k_{B} T\right)$, and noting that $\sinh (x) \approx e^{x} / 2$ for $x \gg 1$, Eq. (33) gives:

$$
f \approx \frac{k_{B} T}{V} \log \left(\frac{\dot{p}}{\dot{p}_{0}}\right)+\frac{\Delta G}{V}
$$

At $T=0 K$, Eq. (39) gives the zero-temperature (effective) flow stress as:

$$
f_{y 0} \approx \frac{\Delta G}{V} .
$$

Eq. (40) clarifies the relationship between the flow stress, $\Delta G_{0}$ and $V$ (Figs 2a and 2c). A larger $\Delta G_{0} / V$ ratio results in a larger apparent yield stress. In other words, a smaller applied stress is needed to activate a larger STZ or a STZ with lower energy barrier. Figs $2 \mathrm{~b}$ and $2 \mathrm{~d}$ also show that materials with larger energy barrier and smaller apparent STZ volume experience larger disorder and consequently larger permanent dilatation, cf. Eq. (6).

The effect of the relaxation parameter $q_{0}$ is illustrated in Figs 3a-3b for a constant strain rate $\dot{\varepsilon}=10^{-5} \mathrm{~s}^{-1}$. In the early stages of deformation (pseudo-elastic deformation), all stress-strain and free volume-strain curves overlap for different values of $q_{0}$. In the softening stage, larger $q_{0}$ (faster structural relaxation) leads to a reduced softening, which corresponds to a smaller increase in free volume. In addition, larger $q_{0}$ causes faster access to steady-state, smaller steady-state free volume and larger steady-state stress. Figs 3c 


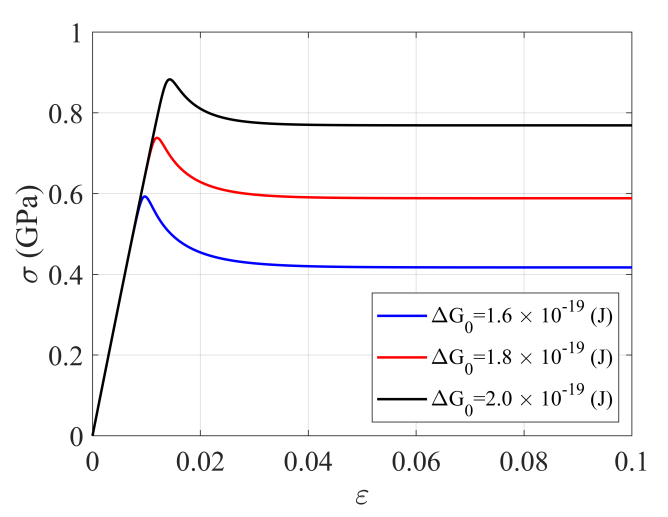

(a)

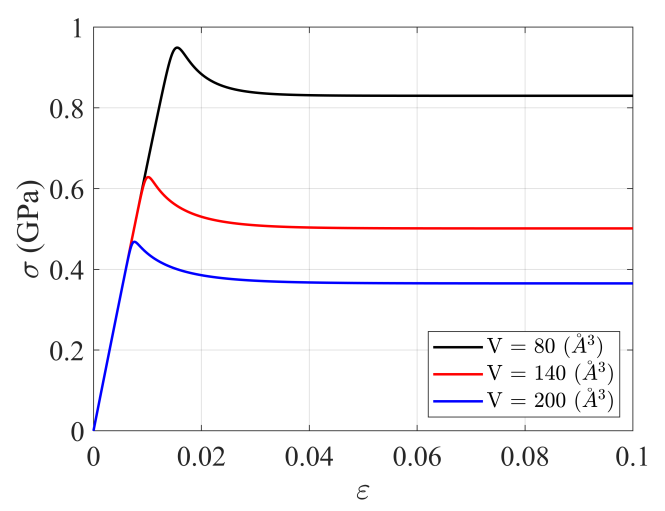

(c)

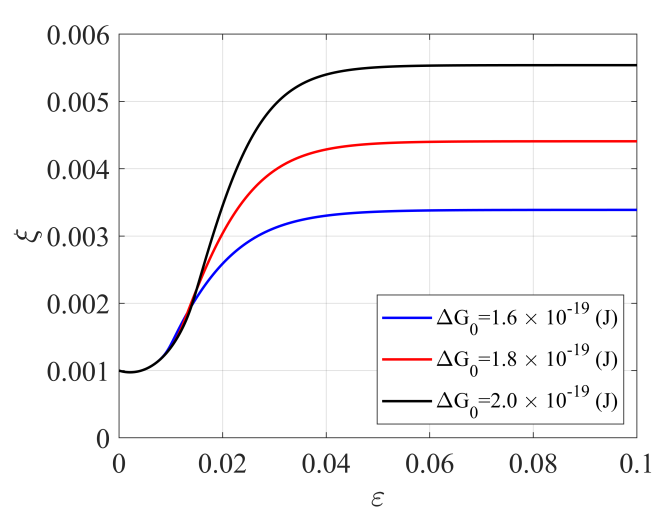

(b)

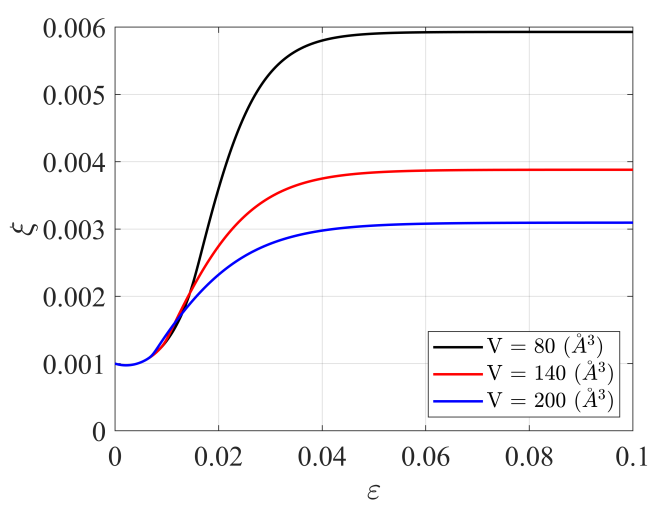

(d)

Figure 2: Effects of energy barrier $\Delta G_{0}$ and apparent STZ volume $V$ on the stress-strain response and free volume evolution in uniaxial tension at rate $\dot{\varepsilon}=10^{-5} \mathrm{~s}^{-1}$.

-3d show the effect of the pressure-sensitivity parameter $b$. According to Eq. (31), the creation of disorder is proportional to $b$. This explains the more pronounced softening 


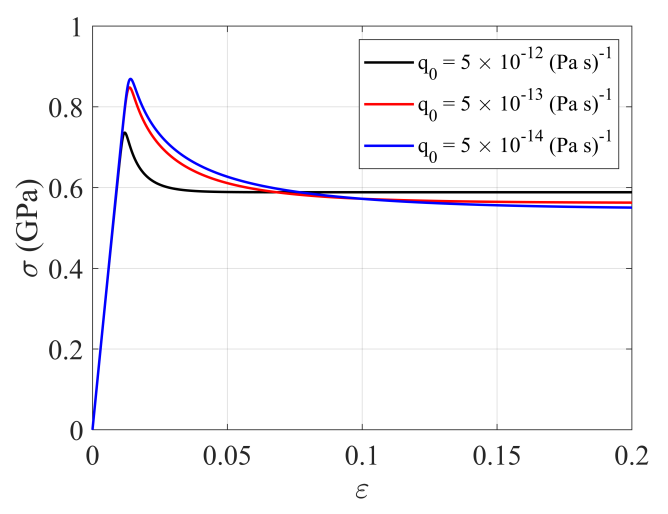

(a)

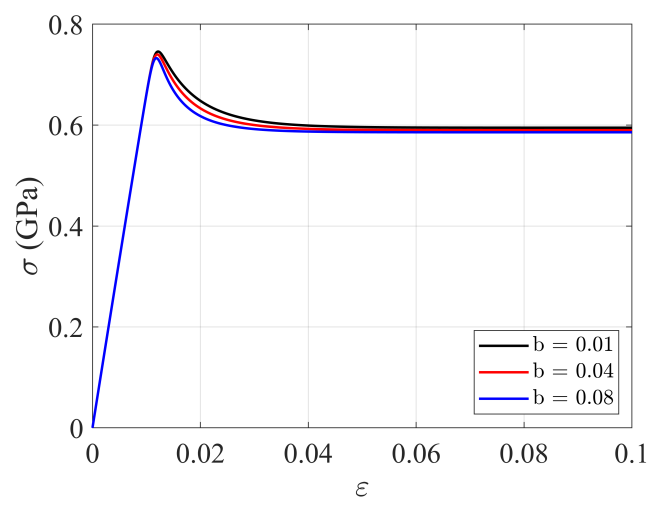

(c)

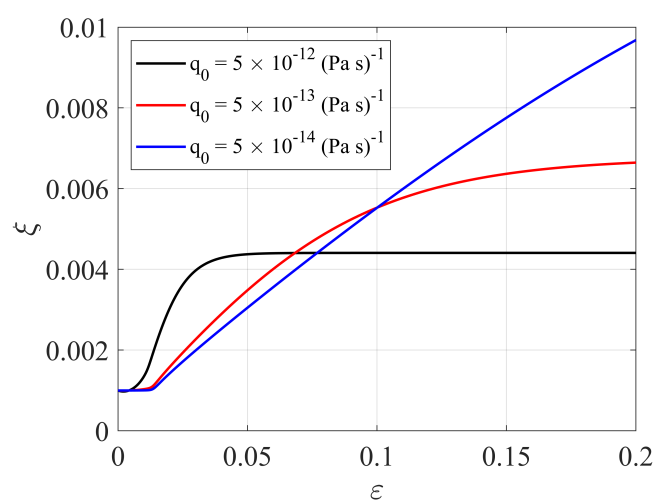

(b)

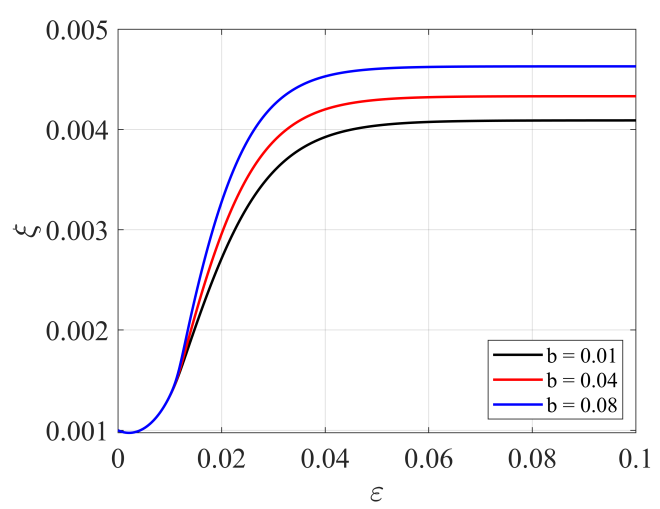

(d)

Figure 3: Effects of structural relaxation rate $q_{0}$ and pressure-sensitivity parameter $b$ on the stress-strain response and free volume evolution in uniaxial tension at rate $\dot{\varepsilon}=10^{-5} \mathrm{~s}^{-1}$. 


\subsection{Cyclic loading}

We now consider uniaxial tension-compression cycles. Material parameters are the same as in the previous section, unless otherwise indicated. A constant strain rate $\dot{\varepsilon}=$ $10^{-3} \mathrm{~s}^{-1}$ is applied in the loading direction. Fig. 4 shows the evolution of stress and free volume during three cycles, for two different values of the relaxation parameter $q_{0}$ : $q_{0}=5 \times 10^{-12}(\mathrm{~Pa} \mathrm{~s})^{-1}($ Figs $4 \mathrm{a}-4 \mathrm{~b})$ and $q_{0}=1 \times 10^{-13}(\mathrm{~Pa} \mathrm{~s})^{-1}($ Figs $4 \mathrm{c}-4 \mathrm{~d})$. Both cases exhibit common features during the first cycle. In the early stages of the first cycle, deformation is mainly elastic and the change in free volume is negligible. As the stress reaches the peak yield stress, plastic deformations and free volume increase sharply, and the stress decreases towards its steady-state value. Upon load reversal, firstly the creation of free volume is zero (elastic unloading) and structural annihilation is the only active process in the evolution of free volume. As the material is further deformed in compression, creation of free volume overcomes annihilation and $\xi$ increases again. Reversing loading again first leads to a decrease in free volume (elastic unloading) followed by increase due to plastic dilatation.

Marked differences are seen between the two cases in subsequent cycles. For faster structural relaxation, $q_{0}=5 \times 10^{-12}(\mathrm{~Pa} \mathrm{~s})^{-1}$ (Fig. 4b), the annihilation rate is sufficiently fast to overcome free volume creation during the compressive portion of the loading. As a result, the free volume is lower in compression. The difference between the first and second cycles in this case is the larger value of $\xi$ in the second cycle, which increases the relaxation driving force $\zeta$ (in absolute value), cf. Eq. (28). In addition, the curves for the second and third cycles (as well as subsequent cycles) overlap, i.e. a steady-state regime is reached. In contrast, for slower structural relaxation, $q_{0}=1 \times 10^{-13}(\mathrm{~Pa} \mathrm{~s})^{-1}$ (Fig. $\left.4 \mathrm{~d}\right)$, structural relaxation does not compensate for free volume creation during plastic loading, and the free volume keeps increasing cycle after cycle.

Regarding the evolution of stress, both cases show a peak in the first cycle, followed by softening due to the increase in free volume (Fig. 4a and Fig. 4c). However, the softening disappears in subsequent cycles, because the considered loading rate does not allow the material to rejuvenate between cycles. In the case of "fast" relaxation (Fig. 4a), the values of stress during the second and third cycles of stress overlap, similar to the free volume value (Fig. 4b). For "slow" relaxation (Fig. 4c), stress cycles do not overlap, since the free volume does not reach the steady-state regime (Fig. 4d). The stress plateau 


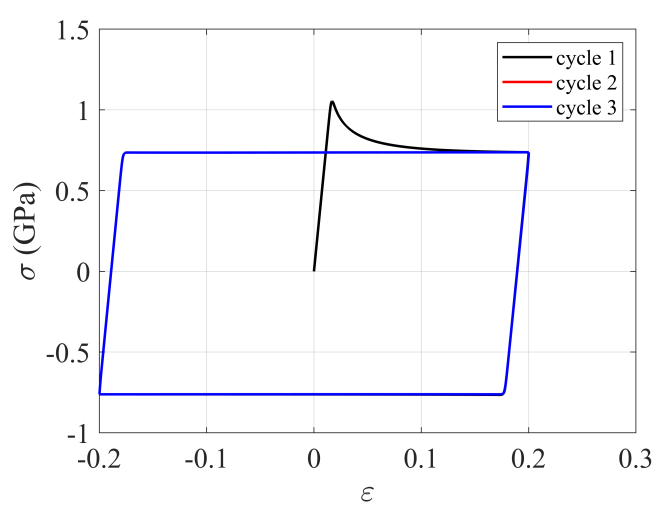

(a)

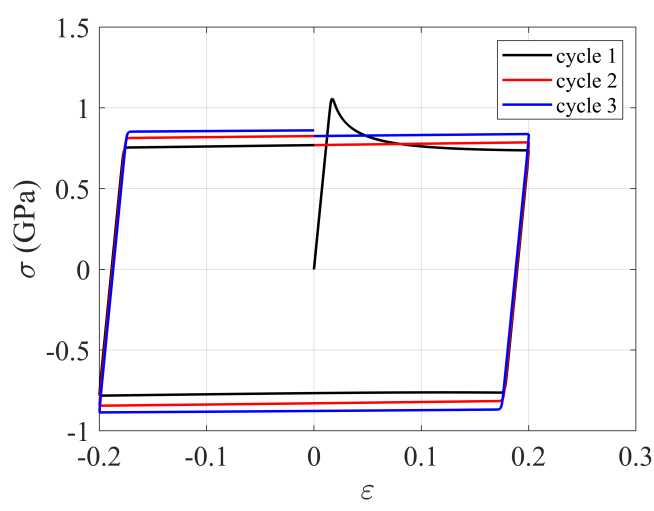

(c)

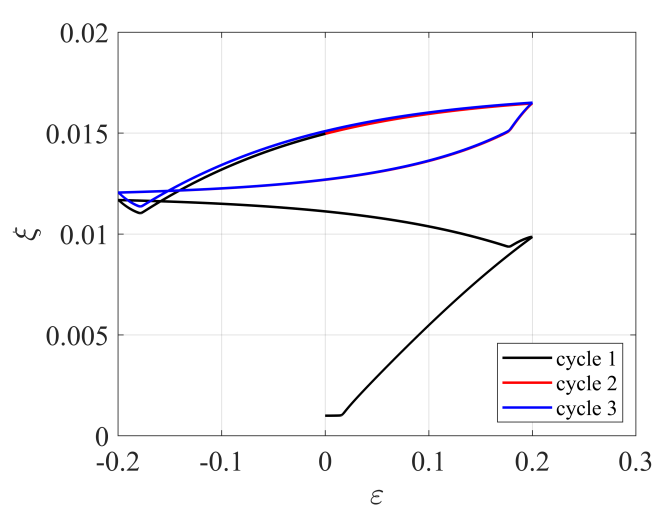

(b)

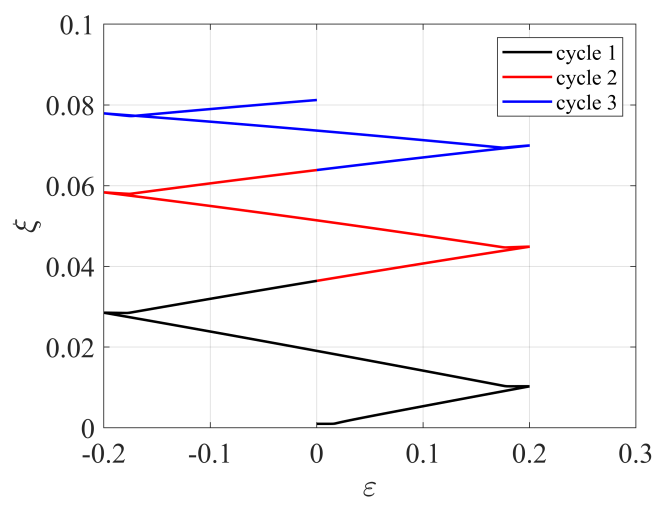

(d)

Figure 4: Uniaxial tension-compression cyclic loading at applied strain rate $|\dot{\varepsilon}|=10^{-3} \mathrm{~s}^{-1}$, for $q_{0}=$ $5 \times 10^{-12}(\mathrm{~Pa} \mathrm{~s})^{-1}(\mathrm{a}-\mathrm{b})$ and $q_{0}=1 \times 10^{-13}(\mathrm{~Pa} \mathrm{~s})^{-1}(\mathrm{c}-\mathrm{d})$, respectively.

value increases with the number of cycles, which seems counter-intuitive given that the free volume decreases the energy barrier for flow (Eq. (34)). There is however a second contribution of free volume in the driving force $\zeta$, Eq. (28), which in turn increases the value of stress needed for flow according to the yield criterion (Eq. (29)). This second contribution is dominating in this case.

\subsection{Free lithiation/delithiation}

We now consider a host subjected to cycles of lithiation and delithiation under no stress. In this case, the yield function $f \leq 0$ at all times, so that $\dot{p}=0$ (Eq. 32) and $\dot{\xi}_{\sigma}=0$ (Eq. (31)). The only contributions to free volume evolution are creation during insertion or extraction of lithium, and structural relaxation. Using Eqs (9), (10) and (37), we have:

$$
\dot{\xi}=\dot{\xi}_{c}+\dot{\xi}_{r}=\frac{\beta \Omega}{J^{c}}|\dot{C}|+q \zeta
$$


where

$$
\zeta=-k^{\prime} \xi=-\frac{k J^{c}}{J} \xi,
$$

and $q=q_{0} \xi$ (Eq. (38)). For prescribed charging/discharging rate $|\dot{C}|$, Eq. (41) constitutes a nonlinear ODE for the free volume as a function of time. We consider lithiation/delithiation cycles between the unlithiated state and a state with concentration $C_{f}$. Let $T_{c h}$ be the charging time needed to reach concentration $C_{f}:|\dot{C}|=C_{f} / T_{c h}$. The free volume at $t=0$ is $\xi_{0}$. The ODE is easily integrated numerically.

Besides the numerical solution, it is useful to consider the analytical solution to a simplified linear case. Neglecting all deformations $\left(J=J^{c} \approx 1\right)$ and assuming linear relaxation kinetics ( $q$ constant), the ODE becomes:

$$
\dot{\xi}=\beta \Omega|\dot{C}|-k q \xi
$$

This expression identifies a structural relaxation time, $T_{r}=(k q)^{-1}$. For a lithiation step between a time $t_{i}$ and current time $t$ under constant (dis)charging, the solution to the linear ODE (43) is readily obtained:

$$
\frac{\xi-\xi_{i}}{\xi_{s s}-\xi_{i}}=1-\exp \left(-\left(t-t_{i}\right) / T_{r}\right)
$$

where $\xi_{i}=\xi\left(t_{i}\right)$ (in particular, $\xi_{i}=\xi_{0}$ when $t_{i}=0$ ), and $\xi_{s s}$ is the steady-state solution:

$$
\xi_{s s}=\beta \Omega|\dot{C}| T_{r}=\beta \Omega C_{f} \frac{T_{r}}{T_{c h}} .
$$

Note that $\beta$ in Eq. (45) takes different values during lithiation and delithiation, according to Eq. (11). The steady-state free volume is proportional to the ratio $T_{r} / T_{c h}$, which illustrates the competition between free volume relaxation and free volume creation during charge/discharge. The steady-state solution can only be reached during a particular lithiation or delithiation step if $T_{r} \ll T_{c h}$.

We illustrate the homogeneous response under free lithiation/delithiation cycles using the full model with the following set of basis parameters: $\beta_{l}=0.003, \beta_{d}=0.005, \Omega=$ $1.36 \times 10^{-29} \mathrm{~m}^{3}$ and $\xi_{0}=0.001$. We take $C_{f}=C_{\text {max }}$, where $C_{\max }$ is estimated from the volume of fully-lithiated silicon: $\Omega C_{\max } \approx 3$. In analogy with the linear case, the structural relaxation time for the nonlinear model is expressed as $T_{r}=(k q)^{-1} \approx\left(k q_{0} \hat{\xi}\right)^{-1}$, where $\hat{\xi}$ is a reference value of free volume, set as $\hat{\xi}=0.005$. We choose $\left(k q_{0}\right)^{-1}=0.05 \mathrm{~h}$, which corresponds to $T_{r}=10 \mathrm{~h}$. 
The evolution of free volume and plastic dilatation with concentration during one lithiation-delithiation cycle are shown in Fig 5 a for various ratios $T_{c h} / T_{r}$. Free volume increases during both lithiation and delithiation. When $T_{c h}<T_{r}$ (fast lithiation), structural relaxation has not time to take place, leading to a pronounced increase in free volume, and hence of plastic dilatation. In contrast, when $T_{c h}>T_{r}$, the increase of free volume saturates as free volume creation and annihilation balance each other. In all these examples, a permanent inelastic volume change remains after one full cycle of lithiationdelithiation, which corresponds to the partial of volume occupied by lithium that was not able to instantaneously recover. These results show that larger plastic dilatation occurs at smaller ratios $T_{c h} / T_{r}$. This is in qualitative agreement with experimental results, which show delithiated $\mathrm{Li}_{x} \mathrm{M}(\mathrm{M}=\mathrm{Si}, \mathrm{Sn}, \mathrm{Ge})$ has a larger volume after delithiation than before lithiation (Ghassemi et al., 2011; McDowell et al., 2013; DeCaluwe et al., 2015; Wang et al., 2015; Chen et al., 2016; Jerliu et al., 2017; Wetjen et al., 2018).

The evolution of $\xi$ during several free lithiation-delithiation cycles for $T_{c h} / T_{r}=0.05$ and $T_{c h} / T_{r}=2$ is shown in Figs. $5 \mathrm{~b}$ and $5 \mathrm{c}$, respectively. Fig. $5 \mathrm{~b}$ reveals that, when $T_{c h}<T_{r}$, the material does not have time to relax and therefore the disorder increases monotonically from one cycle to another. In contrast, when $T_{c h}>T_{r}$ (Fig. 5c), the creation of disorder may overcome the relaxation in early cycles (here, the first two cycles). However, relaxation becomes dominant in subsequent cycles, due to the effect of free volume in the relaxation driving force (Eq. 28). As a result, a steady-state regime is gradually reached where cycles of free volume evolution overlap. Also note that free volume can decrease during lithiation because $\beta_{l}<\beta_{d}$.

Figs. $6 \mathrm{a}$ and $6 \mathrm{~b}$ show the effects of $\beta_{l}$ and $\beta_{d}$ for $T_{c h} / T_{r}=0.1$. Larger values of $\beta_{l}$ result in larger rate of free volume creation during lithium insertion, as expected (Fig. 6a). Similarly, larger values of $\beta_{d}$ result in larger rates of free volume creation during lithium extraction (Fig. 6b).

\section{Comparison to experimental results}

In this section, we use the proposed model to describe experimental results for a-Si thin-film electrodes reported by Bucci et al. (2014) and Pharr et al. (2014). In both sets of experiments, the ratio of charging time to characteristic diffusion time is much larger than unity (see below), and therefore the lithium concentration is assumed homogeneous 


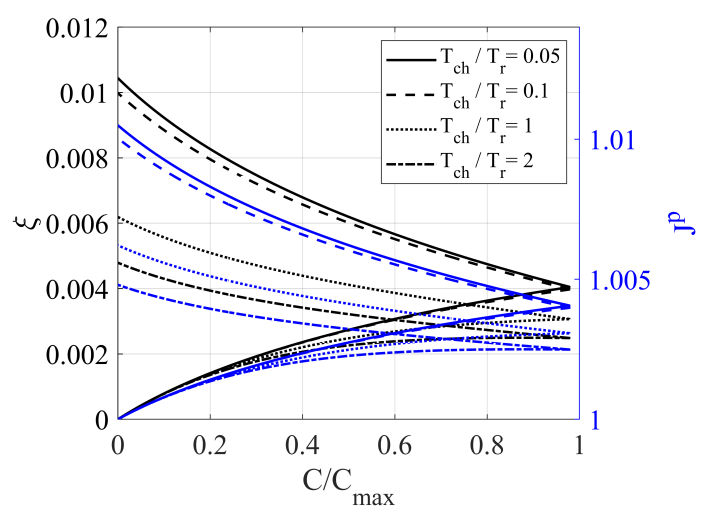

(a)

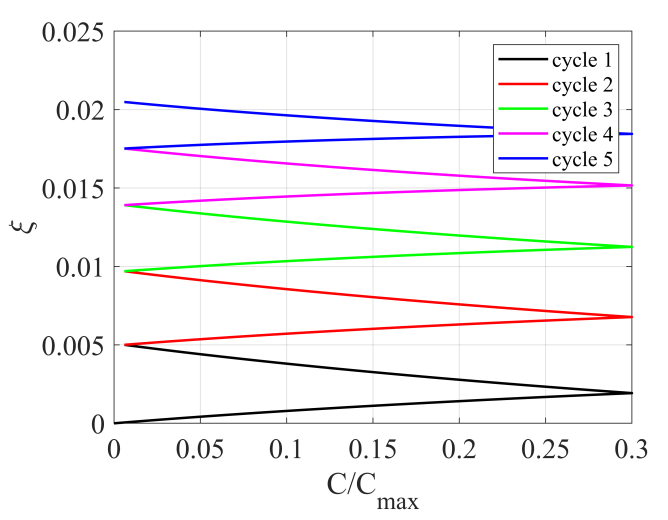

(b)

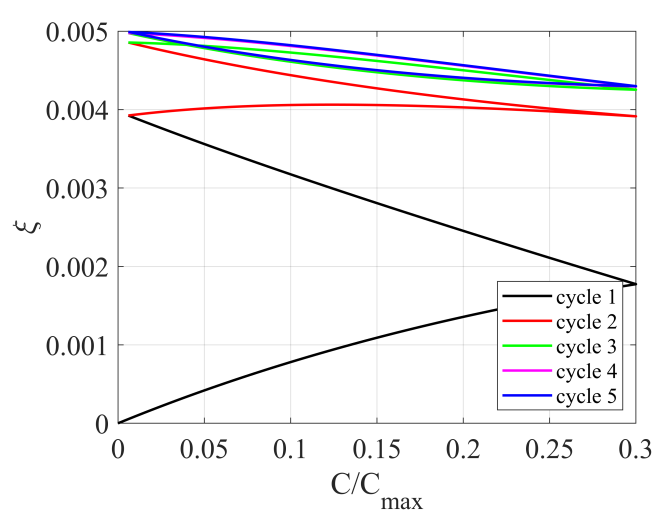

(c)

Figure 5: a) Evolution of free volume and plastic dilatation during free lithiation-delithiation of $\mathrm{Si}$ electrode with different ratios $T_{c h} / T_{r}$. b,c) Evolution of free volume during 5 cycles for $T_{c h} / T_{r}=0.05$ (b) and $T_{c h} / T_{r}=2(\mathrm{c})$.

throughout the film thickness. The state of strain and stress is thus also homogeneous, with zero in-plane strain and zero out-of-plane stress.

As shown in the following, the two sets of experimental results show contrasted responses in terms of rate sensitivity, namely a mostly rate-independent response (Bucci et al., 2014) and a very rate-sensitive response (Pharr et al., 2014). Assuming a homogeneous lithium concentration, we hypothesise that the difference in rate sensitivity is due to different film microstructures resulting from different preparation conditions. In particular, different sputtering pressures were used in the two sets of experiments. It has been previously reported that the sputtering pressure impacts the density, stiffness and strength of sputtered films (van der Rest et al., 2017; Kumari et al., 2018). It is therefore possible that it also affects the rate sensitivity, since rate sensitivity and strength are intrinsically coupled through the micro-mechanisms of plastic deformations in amorphous materials. 


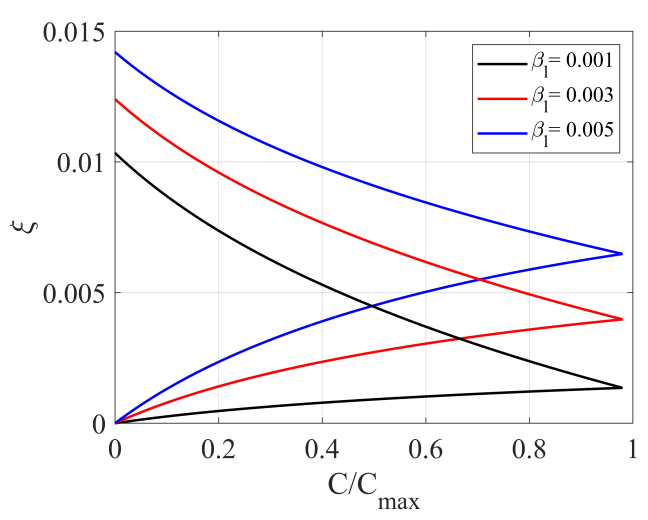

(a)

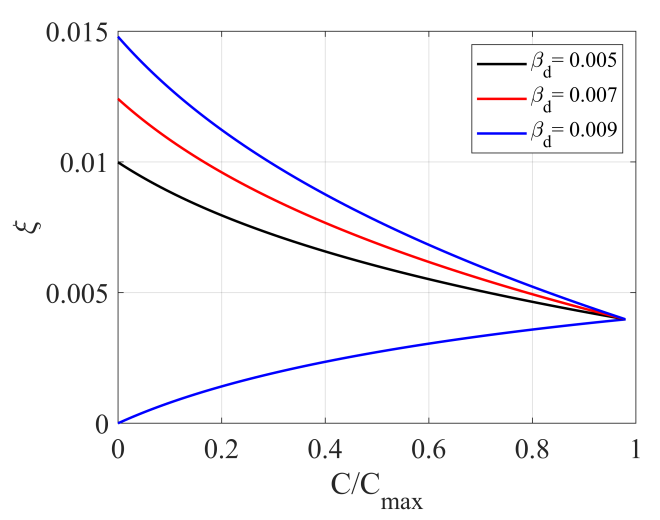

(b)

Figure 6: Evolution of free volume during one free lithiation-delithiation cycle for various values of a) $\beta_{l}$, and b) $\beta_{d}$.

Material parameters used in the numerical model are the following. The Young's modulus was assumed to vary linearly with the lithium concentration. The linear relation was obtained based on operando nanoindentation results obtained by de Vasconcelos et al. (2017). These authors found that the elastic modulus of lithiated amorphous silicon decreases approximately linearly from about $110 \mathrm{GPa}$ to $30 \mathrm{GPa}$ as lithium concentration increases from zero to its maximum value in silicon. Accordingly, we set: $E=110-80 C / C_{\max } \mathrm{GPa}$. Linear relationships for the modulus as a function of concentration ("rules of mixtures") were previously used for alloy-forming electrode materials, including Si, Sn and Al Shenoy et al. (2010); Qi et al. (2014). Poisson's ratio was set to $\nu=0.3$, which is close to experimental value reported by Shenoy et al. (2010) for unlithiated silicon. We further neglected the dependence of Poisson's ratio in lithium concentration. Parameters of the viscoplastic and insertion model used to reproduce each set of experimental data are listed in Table 1. Most of the parameters are identical for both sets. The main difference is the apparent activation volume $V$, which is inversely proportional to the rate-sensitivity: $V \sim \frac{k_{B} T}{\sigma} \frac{\partial(\log \dot{\varepsilon})}{\partial(\log \sigma)}$ (Caillard and Martin, 2003). Rate-insensitive results (Bucci's) are reproduced using a large activation volume, while rate-sensitive results (Pharr's) require a much smaller activation volume.

\subsection{Comparison to experimental data from Bucci et al. (2014)}

Fig. 7a (pink circles) shows experimental mean stress-time curve for a thin-film electrode subjected to three lithiation-delithiation cycles under constant current, with charging times for each cycle respectively given by $C / 25, C / 12.5$ and $C / 8.3$. The diffusion 
Table 1: Parameters used in the numerical simulation of thin-film experiments. Set 1 is used to reproduce the results of Bucci et al. (2014). Set 2 is used to reproduce the results of Pharr et al. (2014).

\begin{tabular}{cccc}
\hline Parameter & Units & Value (Set 1) & Value (Set 2) \\
\hline$V$ & $\AA^{3}$ & 300 & 180 \\
$\Delta G_{\text {lith }}$ & $\mathrm{J}$ & $4 \times 10^{-19}$ & $2.1 \times 10^{-19}$ \\
$\Delta G_{\text {unlith }}$ & $\mathrm{J}$ & $5.3 \times 10^{-19}$ & $2.8 \times 10^{-19}$ \\
$n$ & - & 0.1 & 0.25 \\
$m$ & - & 0.01 & 0.01 \\
$\dot{p}_{0}$ & $\mathrm{~s}^{-1}$ & $5 \times 10^{8}$ & $5 \times 10^{8}$ \\
$b$ & - & 0.055 & 0.055 \\
$k$ & $\mathrm{~Pa}$ & $4 \times 10^{9}$ & $4 \times 10^{9}$ \\
$q_{0}$ & $(\mathrm{~Pa} \mathrm{~s})^{-1}$ & $6 \times 10^{-15}$ & $6 \times 10^{-15}$ \\
$\beta_{l}$ & & 0.001 & 0.001 \\
$\beta_{d}$ & & 0.005 & 0.005 \\
$\xi_{0}$ & - & 0.003 & 0.003 \\
\hline
\end{tabular}

coefficient of lithium into silicon is $D \sim 10^{-16} \mathrm{~m}^{2} / \mathrm{s}$ (Ding et al., 2009), and the film thickness is $h=127 \mathrm{~nm}$, giving a characteristic diffusion time $T_{d}=h^{2} / D$ of about 0.045 $\mathrm{h}$, well below the considered nominal charging times. The experimental data show the usual thin-film behaviour, with a compressive stress during lithiation switching to tension when lithium is removed. Although the rate of lithiation/delithiation is different during these three successive cycles, the stress does not show a significant rate sensitivity. Model predictions are shown in blue in the figure. Best-fit parameters for this experiment are reported in Table 1 as "Set 1". Note that the value of the apparent STZ volume $V$ is $300 \AA^{3}$, which corresponds to the value obtained by (Berla et al., 2015) based on nanoindentation creep experiment.

The most significant discrepancy between numerical and experimental curves occurs during the early stages of the first lithiation, i.e. the elastic part of the response. The slope of the calculated stress-time curve is much higher than the experimentally one. The discrepancy in the elastic regime is attributed to parasitic side reactions and the formation of solid electrolyte interphase (SEI) layer, which are not described by the model. This is however observed in the first lithiation only, after which the model is able to capture 


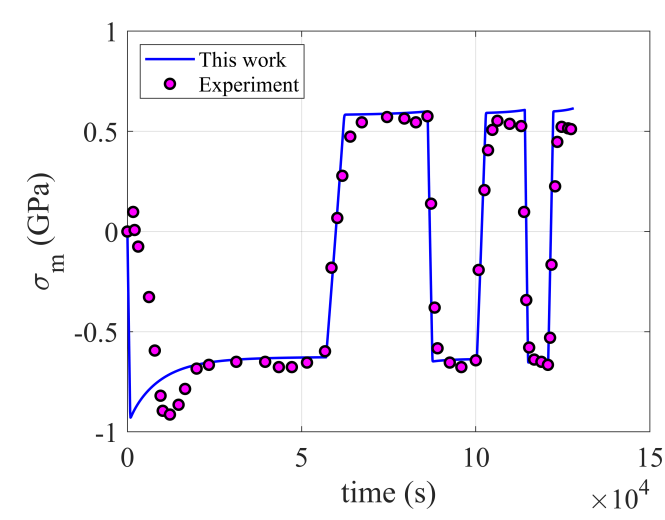

(a)

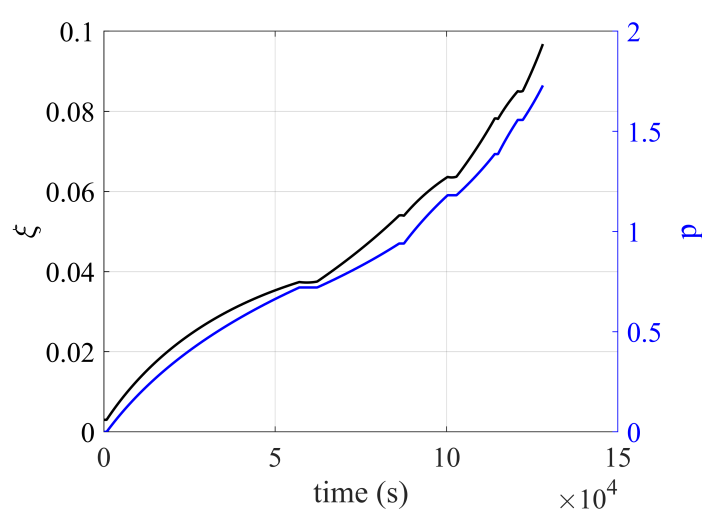

(b)

Figure 7: a) Evolution of mean stress as a function of time in a thin film electrode. Lithiation rates during the first, second and third cycles are $\mathrm{C} / 25, \mathrm{C} / 12.5$ and $\mathrm{C} / 8.3$, respectively. Experimental results are taken from (Bucci et al., 2014). b) Corresponding evolution of free volume and accumulated plastic strain, as predicted by the model.

the elastic and plastic response (i.e. elastic slope and flow stress) and its evolution with charging rate and lithium concentration. In particular, the set of fitting parameters reproduces the weak strain-rate sensitivity.

The evolution of free volume and accumulated plastic strain during these three cycles predicted by the model are depicted in Fig. 7b. Portions of elastic loading are well identified by the plateau values of $p$. The free volume is also approximately constant during elastic loading, because structural relaxation is almost compensated by creation due to lithium insertion/extraction. Overall, free volume creation is strongly correlated to the accumulation of plastic deformation, which follows from the flow rule (31). The rate of free volume creation (black curve) is however larger during delithiation, since we set $\beta_{d}>\beta_{l}$.

\subsection{Comparison to experimental data of Pharr et al. (2014)}

As a second illustration, we consider the experimental measurements of Pharr et al. (2014). The film thickness is $h=100 \mathrm{~nm}$, giving a characteristic diffusion time $T_{d}=0.028$ h. During the first cycle, the film is galvanostatically charged and discharged at rate $\mathrm{C} / 8$. The second cycle starts at a constant $\mathrm{C} / 8$ rate until the concentration reaches about $0.35 C_{\max }$. Subsequently, jumps in charging rates are applied in the following order: $\mathrm{C} / 16$, $\mathrm{C} / 8, \mathrm{C} / 4, \mathrm{C} / 8, \mathrm{C} / 32, \mathrm{C} / 8, \mathrm{C} / 2, \mathrm{C} / 8, \mathrm{C} / 64, \mathrm{C} / 8, \mathrm{C} / 128, \mathrm{C} / 8$. The same sequence is then repeated a second time. The considered charging times are all well above the characteristic 
diffusion time, so that the assumption of homogeneous concentration throughout the film thickness remains reasonable. Fitted model parameters ("Set 2") are reported in Table 1, and the comparison between experimental and numerical results is shown in Fig. 8a.

In contrast to the results of the previous section, experimental results of Pharr et al. (2014) display a significant effect of charging rate on the stress plateau value. Therefore, a smaller apparent activation volume $V$ had to be used in the model in order to obtain good agreement. With this smaller value of activation volume, the model is able to reproduce the experimental results, including the stress jumps due to steps in charging rate during the second cycle, with an increase in lithiation rate resulting in a corresponding increase in the flow stress. The corresponding evolution of free volume and accumulated plastic strain is shown in Fig. 8b. Both free volume and accumulated plastic strain increase when the electrode deforms plastically, with short plateau during elastic loading and unloading. Fluctuations in the free volume evolution associated with lithiation rate jumps during the second cycle show that free volume is more sensitive to rate jumps (via its relaxation mechanism) than the accumulated plastic strain. According to Eq. (6), inelastic volume change in the electrode is $7.7 \%$ at the end of the first cycle.

Similar to Fig. 7a, the model fails to capture the slope of the elastic response during the first lithiation. Furthermore, a significant discrepancy between the model and experimental slope is also seen during the early stages of the second lithiation cycle. One possible reason for the discrepancy could be that the adopted linear relationship for the Young's modulus as a function of lithium concentration is less accurate for the present film at low lithium concentration, possibly due to the different preparation conditions. A second possible origin of the discrepancy could be early anelasticity upon load reversal, similar to the Bauschinger effect. Indeed, early deviation from linearity is clearly seen in the experimental curve during the first delithiation in Fig. 8a, well before the yield point predicted by the model. Likewise, close inspection of the experimental curve during the second load reversal (second lithiation) shows that model and experimental curves are almost parallel in the early (re)lithiation stage. The experimental curve then rapidly diverges from the modelling one. Bauschinger effects have been reported in metallic glasses, and have been attributed to back-stresses generated during the heterogeneous transformation of STZs (Sun et al., 2014; Patinet et al., 2020). It is possible that the observed discrepancy between model and experimental curves during load reversal partly results 


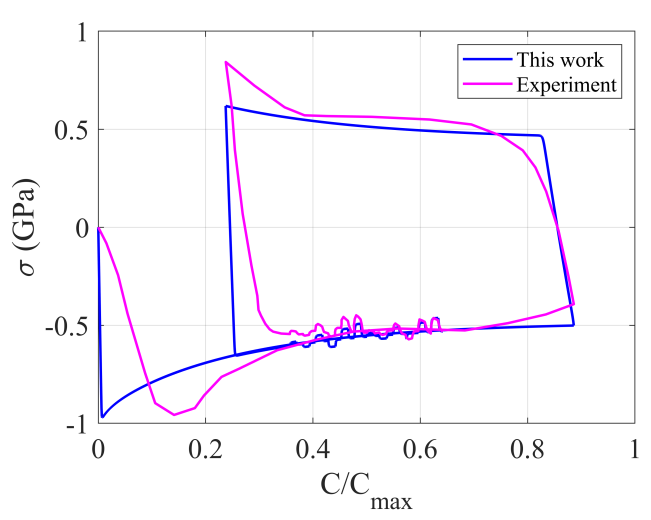

(a)

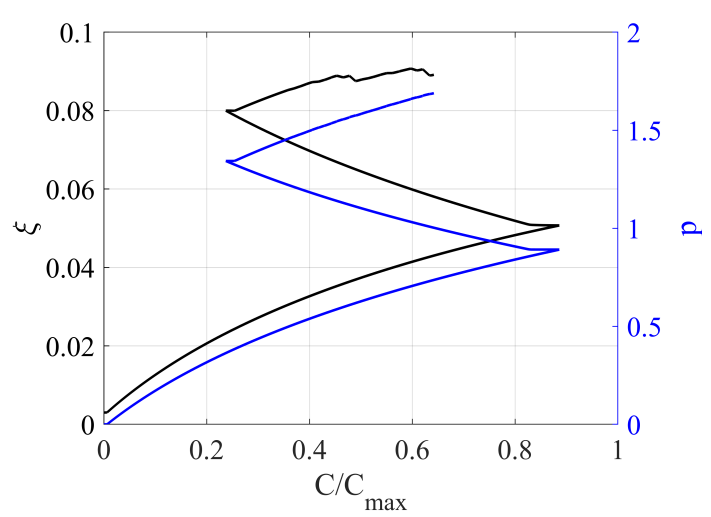

(b)

Figure 8: a) Evolution of stress as a function of concentration in a thin film electrode lithiated at rate C/8. Rate jumps are performed during the second cycle. Experimental results are taken from (Pharr et al., 2014). b) Corresponding evolution of free volume and accumulated plastic strain, as predicted by the model.

from the inability of the model to predict early yielding. Capturing such effect would require incorporating a description of kinematic hardening in the model, which is outside the scope of the present work.

We further compare model predictions obtained with the two sets of parameters listed in Table 1. Fig. 9 shows numerical predictions for thin films lithiated and delithiated at rates $C / 1, C / 8$ and $C / 64$, respectively. The first row shows results obtained with the first set of parameters, and the second row the results obtained with the second set of parameters. As expected, results corresponding to the lower activation volume (Set 2) are more sensitive to charging rate than results obtained with Set 1 parameters. They also show smaller values of flow stress in tension and compression at same charging rates. However, the trends in the evolution of free volume are comparable.

The behaviour of thin-film electrodes during multiple cycles is illustrated in Fig. 10, considering Set 2 parameters and two different charging rates: $\mathrm{C} / 2$ (first row) and $\mathrm{C} / 8$ (second row). Fig. 10a and Fig. 10c show that the values of compression and tension flow stresses slightly increase from one cycle to another. This is again attributed to the driving force for structural relaxation, which increases the magnitude of stress needed for flow. This effect however gradually decreases and saturates after several cycles (the inset in Figs. 10a and 10c). Saturation is reached earlier in the cycling history at low charging rate (large $T_{c h}$ ). Similar to the stress, the increase in free volume tends towards a steadystate, involving a decrease in free volume at the beginning of delithiation as structural 


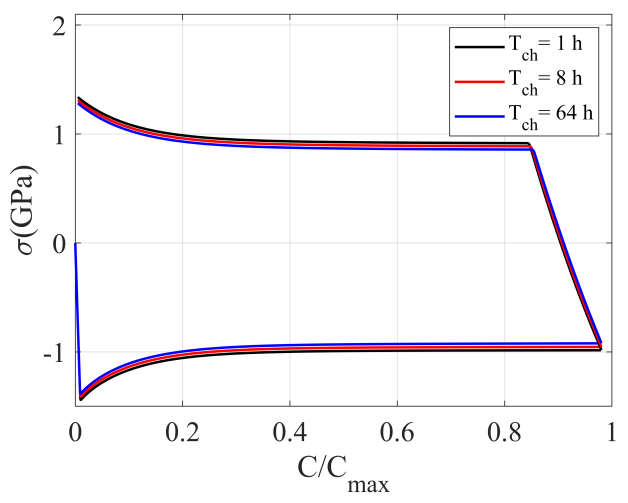

(a)

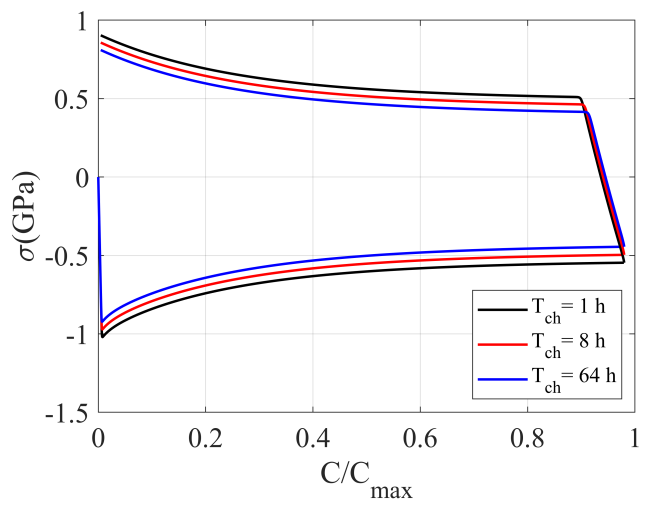

(c)

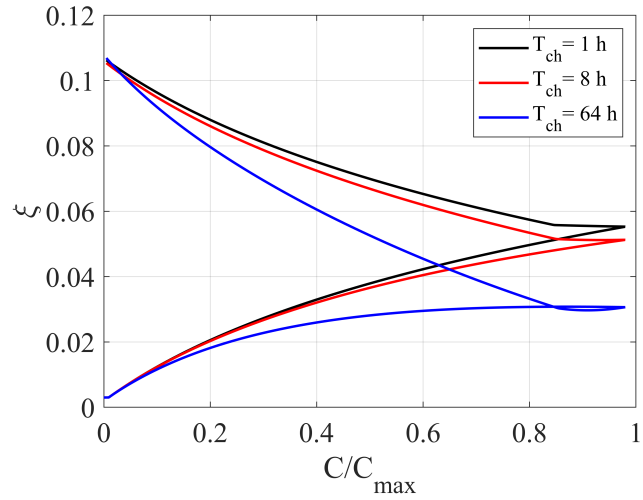

(b)

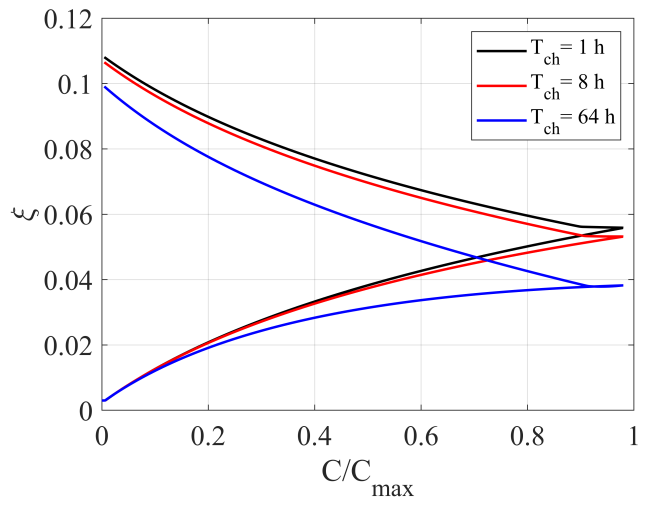

(d)

Figure 9: Predicted lithiation-delithiation cycles for thin films at various charging rates, using Set 1 parameters (a-b) and Set 2 parameters (c-d).

relaxation overcomes free volume creation (Fig. 10d). 


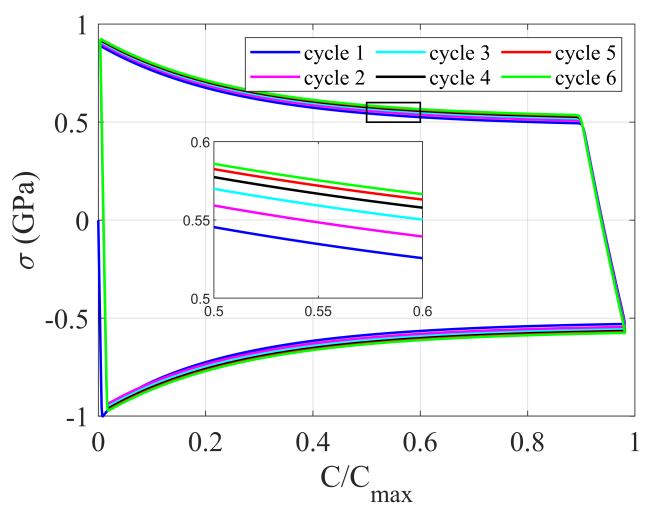

(a)

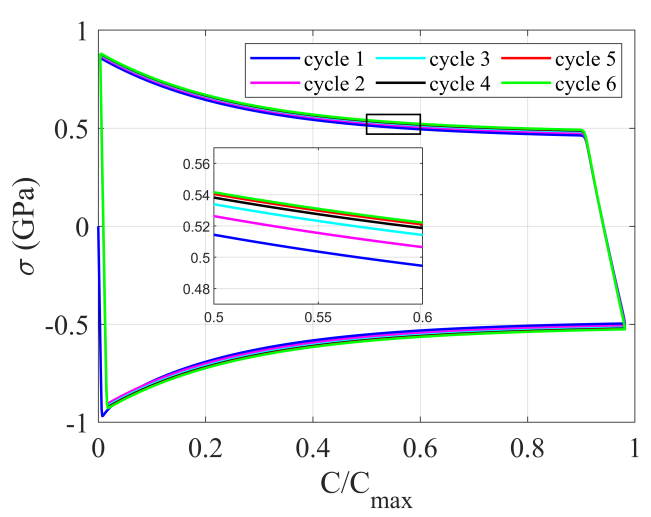

(c)

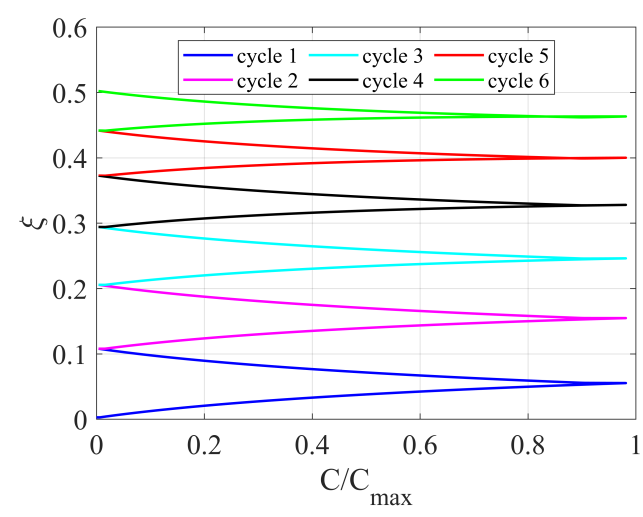

(b)

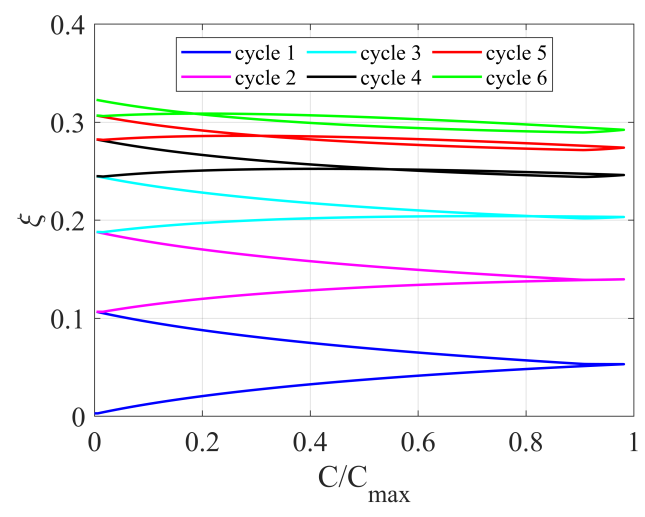

(d)

Figure 10: Predicted lithiation-delithiation cycles for thin films charged at rates a-b) $C / 2$ and c-d) $C / 8$, using Set 2 parameters. 


\section{Conclusions}

We have proposed a constitutive theory that couples large, viscoplastic deformations to the insertion and extraction of lithium in amorphous electrode materials. The theory is based on the concept of Shear Transformation Zones as the carriers of plastic flow in amorphous anode materials, and accounts for microstructural evolution via an internal "free volume" variable. In order to focus on the essential ideas, here we have limited our presentation to homogeneous states described in terms of principal stresses and stretches. Generalization of the theory to a full tensorial representation and investigation of inhomogeneous states are left to future work.

Key features of the model are the following:

1. Rate-dependency: The rate-dependent plastic flow of amorphous electrodes was captured using a STZ-based evolution equation for the plastic multiplier, Eq. (33). Rate sensitivity is primarily governed by the STZ apparent volume $V$, which is inversely proportional to the rate-sensitivity exponent in power-law descriptions of viscoplasticity: $V \sim \frac{k_{B} T}{\sigma} \frac{\partial(\log \dot{\varepsilon})}{\partial(\log \sigma)}$ (Caillard and Martin, 2003). Appropriate selection of the STZ volume allows us to reproduce the rate sensitivity (or lack thereof) displayed in experiments. On the other hand, the stress plateau value is primarily dictated by the ratio $\Delta G / V$. The energy barrier $\Delta G$ was further assumed to decrease with both lithium concentration and free volume.

2. Pressure-dependency: The plasticity model is based on a modified Drucker-Prager yield condition and associative flow rule, Eqs (29)-(31), which predicts asymmetry in tension-compression flow curves. The dilatant part of the plastic strain is kinematically coupled to the creation of free volume (Eq. (6)). As a result, the yield function involves an effective pressure $\zeta$ (Eq. (28)) which depends on both the mean stress and the structural relaxation driving force. In particular, a large value of free volume negatively impacts the yield function due to large structural relaxation driving force. The relative contribution of the effective pressure $\zeta$ to the yield function is governed by the parameter $b$, which also dictates the rate of plasticity-induced free volume creation according to the associative flow rule (31).

3. Structural evolution: The microstructure of the amorphous alloy is described by a free volume variable, which evolves according to three mechanisms: STZ-mediated plastic flow (via the aforementioned kinematic coupling between dilatant plastic 
flow and free volume), insertion/extraction of lithium, and structural relaxation. The parameter $\beta$ governs the rate of free volume created during either insertion or extraction. Here, we assumed that more free volume is created during extraction than during insertion of lithium. The structural relaxation rate is dictated by the ratio $q k$, where $k$ is the energetic parameter quantifying the excess energy associated with free volume - and thus the magnitude of the relaxation driving force - whereas $q$ describes the kinetics of relaxation.

The typical features of amorphous plasticity captured by the model are significant in regard of battery performance. For example, the model predicts a peak in yield stress followed by intrinsic softening before reaching the stress plateau. Values of both the peak stress and stress plateau depend on the deformation rate (Fig. 1), which is itself directly correlated with the lithiation rate when lithiation is constrained (Fig. 9). High stresses generated during fast charging are expected to be detrimental to the battery performance, as they may lead to fatigue and fracture. We also found that the stress and free-volume plateau values are reached earlier in the cycling history when the charging rate is low compared to the structural relaxation rate.

The proposed model is also useful to investigate the issue of nanopore formation in amorphous electrodes of lithium-ion batteries. Previous experimental and numerical investigations have linked the onset of nanopore formation to fast delithiation conditions (Chen and Sieradzki, 2013; Lu et al., 2015, 2016; Kim et al., 2017). Wetjen et al. (2018) further showed that the nanoporous structure reaches a steady state after several cycles. Nanopore build-up not only affects the electrode mechanical integrity, but may also break down the conductive network, leading to isolation of active material and hence capacity fading. While the present model does not explicitly describe pore formation, it may be hypothesised that pore formation results from the accumulation of volumetric inelastic strains associated with free volume. Under this assumption, model predictions are consistent with previous observations for nanopore formation, namely accumulation of free volume during fast (dis)charging, possibly followed by steady state during cyclic loading. The model further suggests that steady-state in free volume - and hence in inelastic volume change - is reached earlier under slow charging conditions. Detailed investigation of the relationships between free volume evolution, dilatant plastic flow, and nanopore formation is suggested as a topic of future work. 


\section{References}

Anand, L., Su, C., 2005. A theory for amorphous viscoplastic materials undergoing finite deformations, with application to metallic glasses. Journal of the Mechanics and Physics of Solids 53, 1362-1396.

Argon, A., 1979. Plastic deformation in metallic glasses. Acta metallurgica 27, 47-58.

Bagheri, A., Arghavani, J., Naghdabadi, R., 2019. On the effects of hydrostatic stress on li diffusion kinetics and stresses in spherical active particles of li-ion battery electrodes. Mechanics of Materials 137, 103134.

Berla, L.A., Lee, S.W., Cui, Y., Nix, W.D., 2015. Mechanical behavior of electrochemically lithiated silicon. Journal of Power Sources 273, 41-51.

Bower, A., Chason, E., Guduru, P., Sheldon, B., 2015. A continuum model of deformation, transport and irreversible changes in atomic structure in amorphous lithium-silicon electrodes. Acta Materialia 98, 229-241.

Bower, A.F., Guduru, P.R., Sethuraman, V.A., 2011. A finite strain model of stress, diffusion, plastic flow, and electrochemical reactions in a lithium-ion half-cell. Journal of the Mechanics and Physics of Solids 59, 804-828.

Brassart, L., Kejie, K., Suo, Z., 2013. Cyclic plasticity and shakedown in high-capacity electrodes of lithium-ion batteries. International Journal of Solids and Structures 50, $1120-1129$.

Brassart, L., Suo, Z., 2012. Reactive flow in large-deformation electrodes of lithium-ion batteries. International Journal of Applied Mechanics 4, 1250023.

Brassart, L., Suo, Z., 2013. Reactive flow in solids. Journal of the Mechanics and Physics of Solids $61,61-77$.

Bucci, G., Nadimpalli, S.P., Sethuraman, V.A., Bower, A.F., Guduru, P.R., 2014. Measurement and modeling of the mechanical and electrochemical response of amorphous si thin film electrodes during cyclic lithiation. Journal of the Mechanics and Physics of Solids 62, 276-294. 
Caillard, D., Martin, J., 2003. Thermally activated mechanisms in crystal plasticity. Pergamon.

Chao, S., Song, Y., Wang, C., Sheu, H., Wu, H., Wu, N., 2011. Study on microstructural deformation of working sn and snsb anode particles for Li-Ion batteries by in situ transmission X-ray microscopy. The Journal of Physical Chemistry C 115, 22040-22047.

Chen, C., Sano, T., Tsuda, T., Ui, K., Oshima, Y., Yamagata, M., Ishikawa, M., Haruta, M., Doi, T., Inaba, M., Kuwabata, S., 2016. In situ scanning electron microscopy of silicon anode reactions in lithium-ion batteries during charge/discharge processes. Scientific Reports 6, 36153.

Chen, Q., Sieradzki, K., 2013. Spontaneous evolution of bicontinuous nanostructures in dealloyed li-based systems. Nature materials 12, 1102-1106.

Cohen, M.H., Turnbull, D., 1959. Molecular transport in liquids and glasses. The Journal of Chemical Physics 31, 1164-1169.

Cui, Z., Gao, F., Cui, Z., Qu, J., 2012. A second nearest-neighbor embedded atom method interatomic potential for li-si alloys. Journal of Power Sources 207, 150-159.

Darbaniyan, F., Yan, X., Sharma, P., 2020. An atomistic perspective on the effect of strain rate and lithium fraction on the mechanical behavior of silicon electrodes. Journal of Applied Mechanics 87, 031011.

De Hey, P., Sietsma, J., Van Den Beukel, A., 1998. Structural disordering in amorphous pd40ni40p20 induced by high temperature deformation. Acta Materialia 46, 5873-5882.

DeCaluwe, S.C., Dhar, B.M., Huang, L., He, Y., Yang, K., Owejan, J.P., Zhao, Y., Talin, A.A., Dura, J.A., Wang, H., 2015. Pore collapse and regrowth in silicon electrodes for rechargeable batteries. Physical Chemistry Chemical Physics 17, 11301-11312.

Demkowicz, M., Argon, A., 2005. Liquidlike atomic environments act as plasticity carriers in amorphous silicon. Physical Review B 72, 245205.

Deshpande, R., Verbrugge, M., Chen, Y.T., Wang, J., Liu, P., 2012. Battery life cycle prediction with coupled chemical degradation and fatigue mechanics. Journal of the Electrochemical Society 159, A1730-A1738. 
Di Leo, C.V., Rejovitzky, E., Anand, L., 2015. Diffusion-deformation theory for amorphous silicon anodes: the role of plastic deformation on electrochemical performance. International Journal of Solids and Structures 67, 283-296.

Ding, J., Cheng, Y.Q., Sheng, H., Asta, M., Ritchie, R.O., Ma, E., 2016. Universal structural parameter to quantitatively predict metallic glass properties. Nature communications 7, 13733.

Ding, N., Xu, J., Yao, Y., Wegner, G., Fang, X., Chen, C., I., L., 2009. Determination of the diffusion coefficient of lithium ions in nano-Si. Solid State Ionics 180, 222-225.

Drucker, D., Prager, W., 1952. Soil mechanics and plastic analysis or limit design. Quarterly of Applied Mathematics 10, 157-165.

Eyring, H., 1936. Viscosity, plasticity, and diffusion as examples of absolute reaction rates. The Journal of chemical physics 4, 283-291.

Falk, M.L., Langer, J.S., 1998. Dynamics of viscoplastic deformation in amorphous solids. Physical Review E 57, 7192.

Fan, F., Huang, S., Yang, H., Raju, M., Datta, D., Shenoy, V., van Duin, A., Zhang, S., Zhu, T., 2013. Mechanical properties of amorphous $\mathrm{Li}_{x} \mathrm{Si}$ alloys: a reactive force field study. Modelling and Simulation in Materials Science and Engineering 21, 074002.

Fan, Z., Ding, J., Li, Q.J., Ma, E., 2017. Correlating the properties of amorphous silicon with its flexibility volume. Physical Review B 95, 144211.

Ghassemi, H., Au, M., Chen, N., Heiden, P.A., Yassar, R.S., 2011. In situ electrochemical lithiation/delithiation observation of individual amorphous si nanorods. ACS nano 5, $7805-7811$.

Hertzberg, B., Benson, J., Yushin, G., 2011. Ex-situ depth-sensing indentation measurements of electrochemically produced si-li alloy films. Electrochemistry Communications $13,818-821$.

Huang, R., Suo, Z., Prevost, J., Nix, W., 2002. Inhomogeneous deformation in metallic glasses. Journal of the Mechanics and Physics of Solids 50, 1011-1027. 
Jerliu, B., Hüger, E., Horisberger, M., Stahn, J., Schmidt, H., 2017. Irreversible lithium storage during lithiation of amorphous silicon thin film electrodes studied by in-situ neutron reflectometry. J. Power Sources 359, 415-421.

Johnson, W.L., Demetriou, M.D., Harmon, J.S., Lind, M.L., Samwer, K., 2007. Rheology and ultrasonic properties of metallic glass-forming liquids: A potential energy landscape perspective. MRS bulletin 32, 644-650.

Karami, A., Nayebi, A., 2020. Kinematic hardening analysis of li-ion battery with concentration-dependent material behaviours under cyclic charging and discharging. Journal of Power Sources 461, 228155.

Khosrownejad, S., Curtin, W., 2016. Model for charge/discharge-rate-dependent plastic flow in amorphous battery materials. Journal of the Mechanics and Physics of Solids $94,167-180$.

Kim, K., Wortman, J., Kim, S.Y., Qi, Y., 2017. Atomistic simulation derived insight on the irreversible structural changes of si electrode during fast and slow delithiation. Nano letters 17, 4330-4338.

Kumari, S., Sharma, S., Mishra, S.K., et al., 2018. Effect of deposition pressure, nitrogen content and substrate temperature on optical and mechanical behavior of nanocomposite al-si-n hard coatings for solar thermal applications. Journal of Materials Engineering and Performance 27, 6729-6736.

Langer, J., 2004. Dynamics of shear-transformation zones in amorphous plasticity: Formulation in terms of an effective disorder temperature. Physical Review E 70, 041502.

Larché, F., Cahn, J., 1978. A nonlinear theory of thermomechanical equilibrium of solids under stress. Acta Metallurgica 26, 1579-1589.

Levitas, V., Attariani, H., 2013. Anisotropic compositional expansion and chemical potential for amorphous lithiated silicon under stress tensor. Scientific Reports 3, 1615.

Levitas, V., Attariani, H., 2014. Anisotropic compositional expansion in elastoplastic materials and corresponding chemical potential: Large-strain formulation and application to amorphous lithiated silicon. Journal of the Mechanics and Physics of Solids 69, $84-111$ 
Li, Y., Mao, W., Zhang, K., Yang, F., 2019a. Analysis of large-deformed electrode of lithium-ion battery: Effects of defect evolution and solid reaction. International Journal of Solids and Structures 170, 1-10.

Li, Y., Mao, W., Zhang, Q., Zhang, K., Yang, F., 2020a. A free volume-based viscoplastic model for amorphous silicon electrode of lithium-ion battery. Journal of The Electrochemical Society 167, 040518.

Li, Y., Zhang, J., Zhang, K., Zheng, B., Yang, F., 2019b. A defect-based viscoplastic model for large-deformed thin film electrode of lithium-ion battery. International Journal of Plasticity 115, 293-306.

Li, Y., Zhang, Q., Zhang, K., Yang, F., 2020b. Coupling effects of self-limiting lithiation, reaction front evolution and free volume evolution on chemical stress in amorphous wire-based electrodes. Journal of Power Sources 457, 228016.

Liu, X.H., Huang, S., Picraux, S.T., Li, J., Zhu, T., Huang, J.Y., 2011. Reversible nanopore formation in ge nanowires during lithiation-delithiation cycling: An in situ transmission electron microscopy study. Nano letters 11, 3991-3997.

Lu, J., Ravichandran, G., Johnson, W.L., 2003. Deformation behavior of the zr41. 2 ti13. 8cu12. 5ni10be22. 5 bulk metallic glass over a wide range of strain-rates and temperatures. Acta materialia 51, 3429-3443.

Lu, X., Bogart, T.D., Gu, M., Wang, C., Korgel, B.A., 2015. In situ tem observations of sn-containing silicon nanowires undergoing reversible pore formation due to fast lithiation/delithiation kinetics. The Journal of Physical Chemistry C 119, 21889-21895.

Lu, X., He, Y., Mao, S.X., Wang, C.m., Korgel, B.A., 2016. Size dependent pore formation in germanium nanowires undergoing reversible delithiation observed by in situ tem. The Journal of Physical Chemistry C 120, 28825-28831.

Ma, E., 2015. Tuning order in disorder. Nature materials 14, 547.

McDowell, M.T., Lee, S.W., Harris, J.T., Korgel, B.A., Wang, C., Nix, W.D., Cui, Y., 2013. In situ tem of two-phase lithiation of amorphous silicon nanospheres. Nano letters $13,758-764$. 
Nadimpalli, S., Sethuraman, V., Dalavi, S., Lucht, B., Chon, M., Shenoy, V., Guduru, P., 2012. Quantifying capacity loss due to solid-electrolyte-interphase layer formation on silicon negative electrodes in lithium-ion batteries. Journal of Power Sources 215, $145-151$.

Patinet, S., Barbot, A., Lerbinger, M., Vandembroucq, D., Lemaître, A., 2020. Origin of the Bauschinger Effect in amorphous solids. Physical Review Letters 124, 205503.

Pharr, M., Suo, Z., Vlassak, J.J., 2013. Measurements of the fracture energy of lithiated silicon electrodes of li-ion batteries. Nano Letters 13, 5570-5577.

Pharr, M., Suo, Z., Vlassak, J.J., 2014. Variation of stress with charging rate due to strain-rate sensitivity of silicon electrodes of li-ion batteries. Journal of Power Sources $270,569-575$.

Qi, Y., Hector Jr, L.G., James, C., Kim, K.J., 2014. Lithium concentration dependent elastic properties of battery electrode materials from first principles calculations. Journal of the Electrochemical Society 161, F3010.

van der Rest, A., Idrissi, H., Henry, F., Favache, A., Schryvers, D., Proost, J., Raskin, J.P., Van Overmeere, Q., Pardoen, T., 2017. Mechanical behavior of ultrathin sputter deposited porous amorphous al2o3 films. Acta Materialia 125, 27-37.

Schuh, C., Hufnagel, T., Ramamurty, U., 2007. Mechanical behavior of amorphous alloys. Acta Materialia 55, 4067-4109.

Schuh, C., Lund, A., 2003. Atomistic basis for the plastic yield criterion of metallic glass. Nature Materials 2, 449-452.

Scrosati, B., Garche, J., 2010. Lithium batteries: Status, prospects and future. Journal of Power Sources 195, 2419-2430.

Sethuraman, V., Srinivasan, V., Bower, A., Guduru, P., 2010a. In situ measurements of stress-potential coupling in lithiated silicon. Journal of the Electrochemical Society 157, A1253-A1261.

Sethuraman, V.A., Chon, M.J., Shimshak, M., Srinivasan, V., Guduru, P.R., 2010b. In situ measurements of stress evolution in silicon thin films during electrochemical lithiation and delithiation. Journal of Power Sources 195, 5062-5066. 
Shenoy, V.B., Johari, P., Qi, Y., 2010. Elastic softening of amorphous and crystalline li-si phases with increasing li concentration: a first-principles study. Journal of Power Sources 195, 6825-6830.

Sitinamaluwa, H., Nerkar, J., Mingchao, W., Zhang, S., Yan, C., 2017. Deformation and failure mechanisms of electrochemically lithiated silicon thin films. RSC Advances 7, $13487-13497$.

Soni, S., Sheldon, B., Xiao, X., Bower, A., Verbrugge, M., 2012. Diffusion mediated lithiation stresses in si thin film electrodes. Journal of the Electrochemical Society 159, A1520-A1527.

Spaepen, F., 1977. A microscopic mechanism for steady state inhomogeneous flow in metallic glasses. Acta Metallurgica 25, 407-415.

Sun, Y., Louzguine-Luzgin, D., Ketov, S., Greer, A., 2014. Pure shear stress reversal on a cu-based bulk metallic glass reveals a bauschinger-type effect. Journal of Alloys and Compounds 615, S75-S78.

Thamburaja, P., Ekambaram, R., 2007. Coupled thermo-mechanical modelling of bulkmetallic glasses: theory, finite-element simulations and experimental verification. Journal of the Mechanics and Physics of Solids 55, 1236-1273.

de Vasconcelos, L.S., Xu, R., Zhao, K., 2017. Operando nanoindentation: a new platform to measure the mechanical properties of electrodes during electrochemical reactions. Journal of The Electrochemical Society 164, A3840-A3847.

Wang, J., Eng, C., Chen-Wiegart, Y.K., Wang, J., 2015. Probing three-dimensional sodiation-desodiation equilibrium in sodium-ion batteries by in situ hard x-ray nanotomography. Nature communications 6, 7496.

Wetjen, M., Solchenbach, S., Pritzl, D., Hou, J., Tileli, V., Gasteiger, H.A., 2018. Morphological changes of silicon nanoparticles and the influence of cutoff potentials in silicon-graphite electrodes. Journal of The Electrochemical Society 165, A1503-A1514.

Yan, X., Gouissem, A., Guduru, P.R., Sharma, P., 2017. Elucidating the atomistic mechanisms underpinning plasticity in li-si nanostructures. Physical Review Materials 1, 055401. 
Yang, Q., Mota, A., Ortiz, M., 2006. A finite-deformation constitutive model of bulk metallic glass plasticity. Computational Mechanics 37, 194-204.

Zhang, S., Zhao, K., Zhu, T., Li, J., 2017. Electrochemomechanical degradation of highcapacity battery electrode materials. Progress in Materials Science 89, 479-521.

Zhang, W.J., 2011. A review of the electrochemical performance of alloy anodes for lithium-ion batteries. Journal of Power Sources 196, 13-24.

Zhao, K., Li, Y., Brassart, L., 2013. Pressure-sensitive plasticity of lithiated silicon in li-ion batteries. Acta Mechanica Sinica 29, 379-387.

Zhao, K., Pharr, M., Cai, S., Vlassak, J., Suo, Z., 2011a. Large plastic deformation in high-capacity lithium-ion batteries caused by charge and discharge. Journal of the American Ceramic Society 94, s226-s235.

Zhao, K., Pharr, M., Vlassak, J., Suo, Z., 2011b. Inelastic hosts as electrodes for highcapacity lithium-ion batteries. Journal of Applied Physics 109.

Zhao, K., Tritsaris, G., Pharr, M., Wang, W., Okeke, O., Suo, Z., Vlassak, J., Kaxiras, E., 2012. Reactive flow in silicon electrodes assisted by the insertion of lithium. Nano Letters 12, 4397-4403.

Zhao, K., Wang, W.L., Gregoire, J., Pharr, M., Suo, Z., Vlassak, J.J., Kaxiras, E., 2011c. Lithium-assisted plastic deformation of silicon electrodes in lithium-ion batteries: a first-principles theoretical study. Nano letters 11, 2962-2967.

Zhao, M., Li, M., 2009. A constitutive theory and modeling on deviation of shear band inclination angles in bulk metallic glasses. Journal of Materials Research 24, 2688-2696.

Zhu, F., Hirata, A., Liu, P., Song, S., Tian, Y., Han, J., Fujita, T., Chen, M., 2017. Correlation between local structure order and spatial heterogeneity in a metallic glass. Physical review letters 119, 215501.

Zhu, F., Song, S., Reddy, K.M., Hirata, A., Chen, M., 2018. Spatial heterogeneity as the structure feature for structure-property relationship of metallic glasses. Nature communications 9, 3965 . 
Zhu, J., Li, W., Wierzbicki, T., Xia, Y., Harding, J., 2019. Deformation and failure of 910 lithium-ion batteries treated as a discrete layered structure. International Journal of $911 \quad$ Plasticity 121, 293-311. 